\title{
Passive Decoupled Multi-Task Controller for Redundant Robots
}

This paper was downloaded from TechRxiv (https://www.techrxiv.org).

\section{LICENSE}

CC BY-NC-SA 4.0

SUBMISSION DATE / POSTED DATE

$16-11-2021 / 19-11-2021$

CITATION

Wu, Xuwei; Ott, Christian; Albu-Schäffer, Alin; Dietrich, Alexander (2021): Passive Decoupled Multi-Task Controller for Redundant Robots. TechRxiv. Preprint. https://doi.org/10.36227/techrxiv.17025314.v1

DOI 


\title{
Passive Decoupled Multi-Task Controller for Redundant Robots
}

\author{
Xuwei Wu, Christian Ott, Alin Albu-Schäffer, and Alexander Dietrich
}

\begin{abstract}
Kinematic redundancy in robots makes it possible to execute several control tasks simultaneously. As some tasks are usually more important than others, it is reasonable to dynamically decouple them in order to ensure their execution in a hierarchical way or even without any interference at all. The most widely used technique is to decouple the system by feedback linearization. However, that requires to actively shape the inertia and consequently modify the natural dynamics of the robot. Here we propose a passivity-based multi-task tracking controller that preserves these inertial properties but fully compensates for taskspace cross-couplings using external force feedback. Additionally, three formal proofs are provided: uniform exponential stability for trajectory tracking, passivity during physical interaction, and input-to-state-stability. The controller is validated in simulations and experiments and directly compared with the hierarchical PD+ approach and the feedback linearization. The proposed approach is well suited for safe physical human-robot interaction and dynamic trajectory tracking if measurements or estimations of the external forces are available.
\end{abstract}

Index Terms-Multi-task control, nonlinear systems, stability, trajectory tracking, physical interaction, redundant robots.

\section{INTRODUCTION}

D RIVEN by the development of more complex robotic systems such as humanoids or service robots with mobile platforms, much research has focused on simultaneous control of multiple objectives in task space to exploit the available kinematic redundancy of the system. As exemplified in Fig. 1, the kinematically redundant robot has more degrees of freedom (DOF) than necessary with respect to the end-effector task, and additional tasks can be performed in parallel by exploiting the remaining DOF. To make sure that safety-critical objectives like collision avoidance can be achieved without any compromise, a task hierarchy is usually required to stack the tasks in accordance with prescribed priorities. However, this prioritized multi-task control strategy poses significant challenges in terms of ensuring compliant behavior in physical interaction with the environment, while preserving control accuracy of trajectory tracking in free motion.

To implement the task hierarchy, a common approach is to decouple the total task space by means of null-space projections [1]-[3]. Thanks to the strict prioritization, one can ensure that control actions for lower-priority tasks do not interfere with the execution of higher-priority ones. An alternative way that has attracted much interest recently is to resolve the redundancy by solving hierarchical quadratic programs (QP) [4]-[8]. When weighting strategies are used [4], [7], [8], only soft prioritization can be realized, which can lead to a

The authors are with the Institute of Robotics and Mechatronics, German Aerospace Center (DLR), 82234 Wessling, Germany Contact: xuwei. wuddr. de.

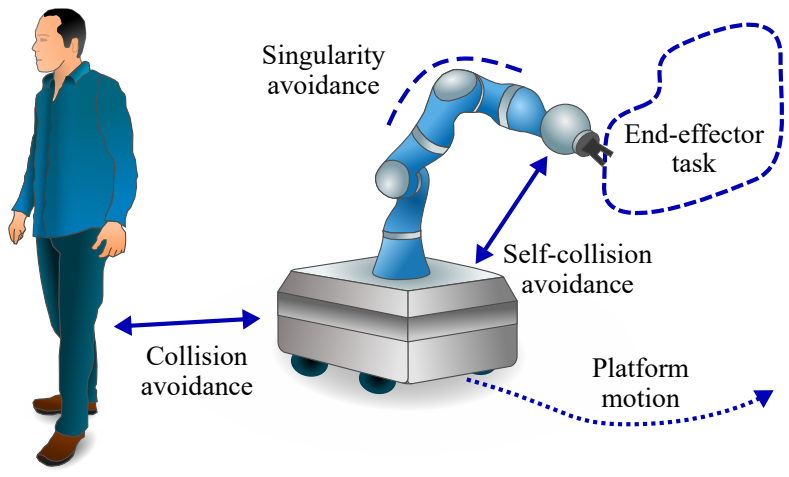

Fig. 1. A motivational example for multi-task control in kinematically redundant robots.

compromise among task objectives regardless of the specified priorities. These QP-based approaches feature the advantage of explicitly incorporating inequality constraints into the task hierarchy, but because of that, they also have the drawback of providing sufficiently analyzed stability properties-a notable recent exception is [7]. For robotic applications where physical interaction with the environment and humans cannot be excluded, it is essential to require the controlled robots to have analytically provable stability properties.

One of the most well-known projection-based approaches with strict prioritization is the Operational Space Formulation (OSF) [1], which has been extended in [9], [10] to allow application of task hierarchies with an arbitrary number of priority levels. In OSF, the desired linear dynamics in task space is characterized by the classical impedance control [11] in order to regulate the robot's interactive behavior with the environment. To implement this, feedback linearization is performed on all priority levels, while an inertial decoupling across the hierarchies is automatically achieved by means of dynamically consistent null-space projectors [3]. Moreover, it has been shown in [12] that the OSF approach can be seen as equivalent to an inverse-dynamics-based approach such as [13]. Due to the linear, fully decoupled closed-loop dynamics, exponential stability of trajectory tracking can be straightforwardly concluded, and the convergence rate can be explicitly set through pole placement. However, the complete cancellation of the natural nonlinear dynamics makes this family of feedback-linearization-based approaches sensitive to modeling errors and parameter variations. In addition, to achieve the desired impedance, knowledge of external forces/torques is typically required.

On the other hand, a set of passivity-based hierarchical approaches have adopted the concept of compliance control [14]. 
It can be regarded as a variation of impedance control while keeping the nonlinear inertial properties in task space, i.e., no inertia shaping is performed as opposed to the feedback linearization. In [15], [16] the regulation problem has been addressed for an arbitrary number of priority levels. Recently, a hierarchical PD+ tracking controller (HPD+) within this framework was proposed in [17], providing uniform asymptotic stability (UAS). Due to the preservation of natural taskspace inertias, all mentioned passivity-based approaches do not rely on the feedback of external forces/torques to achieve the desired impedance behavior. Furthermore, they offer better robustness properties against unmodeled dynamics, and they can also reduce the risk of exceeding feasible actuator limits, especially when the robot undergoes dramatic changes of joint configuration [17]. Nevertheless, exponential stability cannot be shown in general, as a result of remaining couplings in the closed loop.

Among the hierarchical controllers that feature an arbitrary number of priority levels, only a few have provided stability properties with mathematically rigorous proofs. For multi-objective inverse kinematics control, a formal proof of asymptotic stability for the regulation problem has been conducted in [18]. Based on Lyapunov's indirect method, exponential stability of the optimization-based approach with soft prioritization has been shown in [7], but the considered hierarchies can merely contain equality constraints. In [19], UAS of the multi-task tracking control with a controllerobserver structure has been proved. However, the feedback gains have to fulfill some configuration-dependent conditions for a valid proof. Moreover, desired impedance behavior can only be specified on the least-priority level in the null space.

In this article, we propose a passive decoupled multi-task controller with strict prioritization that can suitably handle physical interaction with desired contact stiffness as well as trajectory tracking with advanced performance. It combines the advantages of both the HPD+ approach and the classical feedback linearization in two major aspects. First, the crosscouplings of task-space kinematics and external forces are fully compensated, which allows to conclude the stronger property of uniform exponential stability (UES) and improves the physical interaction behavior. Second, the natural inertial properties of the nonlinear task-space dynamics are preserved, leading to superior robustness inherited from passivity-based approaches. Formal proofs are provided for UES in free motion, passivity during physical interaction, and input-to-statestability (ISS) under bounded interaction forces. To facilitate the stability analysis, a strict Lyapunov function for the overall closed loop is constructed by using the cross-term technique [20]-[24]. The theoretical results are validated in simulations through a planar manipulator and in experiments on a KUKA LWR IV+ with torque control interface, while practical comparisons with the state of the art are provided. Summarized, the main contributions of our multi-task controller include:

- Preserved natural inertia for high robustness;

- Full compensation for dynamic cross-couplings for high performance and safe physical interaction;

- Formal proofs of UES and ISS for trajectory tracking, and proof of passivity for physical interaction;
- Experimental validation of the proposed approach and direct comparison with state-of-the-art methods.

The rest of the article is organized as follows. Section II recalls the fundamentals of inertially decoupled task-space dynamics and the HPD+ control scheme. The control design is then derived in Section III, which also provides a comparative analysis of the proposed approach and the classical feedback linearization. In Section IV, formal proofs of stability and passivity properties are conducted. To validate the theoretical results, Section V and Section VI report simulations and experiments that directly compare the approach with the state of the art. Finally, Section VII concludes this article.

\section{FUndAMENTALS AND PRELIMINARIES}

\section{A. Notations}

Throughout this paper, $|\cdot|$ denotes the absolute value of a real number; $\|\cdot\|$ stands for the Euclidean norm of a vector and the induced norm of a matrix; $\lambda_{\mathrm{m}}(\boldsymbol{A})$ and $\lambda_{\mathrm{M}}(\boldsymbol{A})$ denote the minimum and maximum eigenvalue of the matrix $\boldsymbol{A}$, respectively; we use $\boldsymbol{A} \succ 0$ to indicate that $\boldsymbol{A}$ is positive definite. Note that if $\boldsymbol{A}$ is symmetric and $\boldsymbol{A} \succ 0$, then $\|\boldsymbol{A}\|=\lambda_{\mathrm{M}}(\boldsymbol{A})$ and $\left\|\boldsymbol{A}^{-1}\right\|=1 / \lambda_{\mathrm{m}}(\boldsymbol{A})$. The identity matrices are denoted by $\boldsymbol{I}$ and zero vectors/matrices by $\mathbf{0}$, with their dimensions depending on the context. A diagonal matrix with diagonal entries $\left\{a_{i}\right\}$ is denoted by $\operatorname{diag}\left\{a_{i}\right\}$ and a block diagonal matrix with diagonal blocks $\left\{\boldsymbol{A}_{i}\right\}$ is represented by $\operatorname{diag}\left\{\boldsymbol{A}_{i}\right\}$. The notation $\prod_{i=1}^{n} \boldsymbol{A}_{i}$ represents the left matrix multiplication of a sequence of matrices $\left\{\boldsymbol{A}_{i}\right\}$ with the same dimensions. A continuous function $\gamma: \mathbb{R}_{>0} \rightarrow \mathbb{R}_{>0}$ is of class $\mathcal{K}$, if it is strictly increasing and $\gamma(0)=0$; $\mathbf{A}$ continuous function $\beta: \mathbb{R}_{\geq 0} \times \mathbb{R}_{\geq 0} \rightarrow \mathbb{R}_{\geq 0}$ is of class $\mathcal{K} \mathcal{L}$, if for each fixed $t \geq 0, \beta(s, t) \in \mathcal{K}$ w. r. t. $s$ and, for each fixed $s \geq 0, \beta(s, t) \rightarrow 0$ as $t \rightarrow \infty$.

\section{B. Robot Dynamics}

The joint-space dynamics of a fully-actuated, rigid-link robot with $n$ DOF can be written as:

$$
\boldsymbol{M}(\boldsymbol{q}) \ddot{\boldsymbol{q}}+\boldsymbol{C}(\boldsymbol{q}, \dot{\boldsymbol{q}}) \dot{\boldsymbol{q}}+\boldsymbol{g}(\boldsymbol{q})=\boldsymbol{\tau}+\boldsymbol{\tau}^{\mathrm{ext}}
$$

where $\boldsymbol{q}, \dot{\boldsymbol{q}}, \ddot{\boldsymbol{q}} \in \mathbb{R}^{n}$ are the joint positions, velocities, and accelerations, respectively; $\boldsymbol{M}(\boldsymbol{q}) \in \mathbb{R}^{n \times n}$ is the symmetric and positive definite inertia matrix; $\boldsymbol{C}(\boldsymbol{q}, \dot{\boldsymbol{q}}) \in \mathbb{R}^{n \times n}$ is the Coriolis/centrifugal matrix; $\boldsymbol{g}(\boldsymbol{q}) \in \mathbb{R}^{n}$ is generalized gravitational force. The motor forces/torques $\tau \in \mathbb{R}^{n}$ and the generalized external force $\tau^{\text {ext }} \in \mathbb{R}^{n}$ are inputs to the system. We make two standard assumptions associated with the dynamics (1):

Assumption 1: $\boldsymbol{M}(\boldsymbol{q})$ and $\boldsymbol{C}(\boldsymbol{q}, \dot{\boldsymbol{q}})$ are uniformly bounded for all possible $\boldsymbol{q}$. In addition, $\boldsymbol{C}(\boldsymbol{q}, \dot{\boldsymbol{q}})$ is linear in $\dot{\boldsymbol{q}}$.

Assumption 2: The matrix $\dot{\boldsymbol{M}}(\boldsymbol{q}, \dot{\boldsymbol{q}})-2 \boldsymbol{C}(\boldsymbol{q}, \dot{\boldsymbol{q}})$ is skewsymmetric [23].

Remark 1: In [25], [26] a complete classification of robot manipulators that satisfy Assumption 1 is provided, which includes, for instance, any robot that features only revolute or only prismatic joints. Assumption 2 holds provided that $\boldsymbol{C}(\boldsymbol{q}, \dot{\boldsymbol{q}})$ is properly defined, e. g., as in [27], [28]. 


\section{Task Hierarchy and Task-Space Definitions}

The task hierarchy to be considered consists of $r>1$ levels. The individual task priorities are specified in descending order with an increasing level index from $i=1$ to $i=r$. Each task $\boldsymbol{x}_{i}$ is defined via a differentiable function $\boldsymbol{f}_{i}: \mathbb{R}^{n} \rightarrow \mathbb{R}^{m_{i}}$ :

$$
\boldsymbol{x}_{i}:=\boldsymbol{f}_{i}(\boldsymbol{q})
$$

with the respective task dimension $m_{i}<n$. The corresponding task-space velocity $\dot{\boldsymbol{x}}_{i}$ is given by

$$
\dot{\boldsymbol{x}}_{i}=\boldsymbol{J}_{i}(\boldsymbol{q}) \dot{\boldsymbol{q}}
$$

where $\boldsymbol{J}_{i}(\boldsymbol{q}):=\partial \boldsymbol{f}_{i}(\boldsymbol{q}) / \partial \boldsymbol{q} \in \mathbb{R}^{m_{i} \times n}$ denotes the Jacobian matrix of the $i$-th task. The augmented Jacobian matrix [2] is defined as

$$
\boldsymbol{J}(\boldsymbol{q}):=\left[\begin{array}{lll}
\boldsymbol{J}_{1}^{T}(\boldsymbol{q}) & \ldots & \boldsymbol{J}_{r}^{T}(\boldsymbol{q})
\end{array}\right]^{T} \in \mathbb{R}^{\sum_{i=1}^{r} m_{i} \times n},
$$

and the stacked task-space velocity vector $\dot{\boldsymbol{x}} \in \mathbb{R}^{\sum_{i=1}^{r} m_{i}}$ is

$$
\dot{\boldsymbol{x}}:=\left[\begin{array}{lll}
\dot{\boldsymbol{x}}_{1}^{T} & \ldots & \dot{\boldsymbol{x}}_{r}^{T}
\end{array}\right]^{T}=\boldsymbol{J}(\boldsymbol{q}) \dot{\boldsymbol{q}} .
$$

With regard to the task hierarchy, we assume:

Assumption 3: For all levels $i=1 \ldots r, \boldsymbol{f}_{i}$ is continuously differentiable in $\boldsymbol{q}$, and $\boldsymbol{J}_{i}(\boldsymbol{q})$ is uniformly bounded in $\boldsymbol{q}$.

Assumption 4: All tasks are free of singularities in the considered workspace.

Assumption 5: All tasks are simultaneously independent ${ }^{1}$ from each other and span the state space as a whole, i.e., $\sum_{i=1}^{r} m_{i}=n$.

Remark 2: Assumption 3 is commonly made, although sometimes implicitly, in the literature of task-space control, e. g., [18], [29]-[31]; if $\boldsymbol{f}_{i}(\boldsymbol{q})$ represents a forward kinematics mapping, then the boundedness condition of $\boldsymbol{J}_{i}(\boldsymbol{q})$ is satisfied under the same conditions required by Assumption 1 .

Under Assumptions 4 and $5, \boldsymbol{J}_{i}(\boldsymbol{q})$ is of full row-rank for all levels $i=1 \ldots r$, and $\boldsymbol{J}(\boldsymbol{q}) \in \mathbb{R}^{n \times n}$ is invertible ${ }^{2}$. The generalized task-space external forces are defined as

$$
\boldsymbol{F}_{\dot{\boldsymbol{x}}}^{\mathrm{ext}}:=\boldsymbol{J}(\boldsymbol{q})^{-T} \boldsymbol{\tau}^{\mathrm{ext}}=\left[\begin{array}{lll}
\boldsymbol{F}_{\dot{\boldsymbol{x}}_{1}}^{\mathrm{ext}, T} & \ldots & \boldsymbol{F}_{\dot{\boldsymbol{x}}_{r}}^{\mathrm{ext}, T}
\end{array}\right]^{T}
$$

where $\boldsymbol{F}_{\dot{\boldsymbol{x}}_{i}}^{\text {ext }} \in \mathbb{R}^{m_{i}}$ denotes the force/torque component on the hierarchy level $i$.

As control objective for each task, a reference trajectory $\boldsymbol{x}_{i, \mathrm{des}}(t) \in \mathbb{R}^{m_{i}}$ is specified, which is supposed to be twice continuously differentiable and uniformly bounded in $t$. The vector $\boldsymbol{x}_{\mathrm{des}}(t):=\left[\begin{array}{llll}\boldsymbol{x}_{i, \mathrm{des}}^{T}(t) & \ldots & \boldsymbol{x}_{r, \mathrm{des}}^{T}(t)\end{array}\right]^{T} \in \mathbb{R}^{n}$ stacks all desired task-space positions down to the lowest level. The individual task-space position, velocity, and acceleration errors are defined as $\tilde{\boldsymbol{x}}_{i}:=\boldsymbol{x}_{i}-\boldsymbol{x}_{i, \mathrm{des}}(t), \dot{\tilde{\boldsymbol{x}}}_{i}:=\dot{\boldsymbol{x}}_{i}-\dot{\boldsymbol{x}}_{i, \mathrm{des}}(t)$, and $\ddot{\tilde{\boldsymbol{x}}}_{i}:=\ddot{\boldsymbol{x}}_{i}-\ddot{\boldsymbol{x}}_{i \text {,des }}(t)$, respectively. Correspondingly, their stacked versions are denoted by $\tilde{\boldsymbol{x}}, \dot{\tilde{\boldsymbol{x}}}$, and $\ddot{\tilde{\boldsymbol{x}}}$, analog to (5).

\footnotetext{
${ }^{1} \mathrm{~A}$ rigorous definition of the independency condition between two tasks can be found in [18].

${ }^{2}$ The minimum singular value of $\boldsymbol{J}(\boldsymbol{q})$ is assumed to be greater than a known positive constant $\sigma$.
}

\section{Hierarchy-Consistent Task-Space Definitions}

In order to implement the prescribed priorities of the task hierarchy, the prioritized Jacobian matrices

$$
\overline{\boldsymbol{J}}_{i}(\boldsymbol{q}):=\boldsymbol{J}_{i}(\boldsymbol{q}) \boldsymbol{N}_{i}(\boldsymbol{q})^{T} \in \mathbb{R}^{m_{i} \times n}
$$

are utilized for all levels $i=1 \ldots r$. Herein, the null-space projectors $\boldsymbol{N}_{i}(\boldsymbol{q}) \in \mathbb{R}^{n \times n}$ are supposed to satisfy the dynamic consistency condition [1], [32]

$$
\boldsymbol{J}_{j}(\boldsymbol{q}) \boldsymbol{M}(\boldsymbol{q})^{-1} \boldsymbol{N}_{i}(\boldsymbol{q})=\mathbf{0}, \quad \forall j<i \leq r,
$$

leading to the inertially decoupled task-space dynamics as will be shown in Section II-E. One can compute $\boldsymbol{N}_{i}(\boldsymbol{q})$ recursively by [33], [10]

$$
\begin{aligned}
\boldsymbol{N}_{1} & =\boldsymbol{I} \\
\overline{\boldsymbol{J}}_{1}(\boldsymbol{q}) & =\boldsymbol{J}_{1}(\boldsymbol{q}) \\
\boldsymbol{N}_{i}(\boldsymbol{q}) & =\boldsymbol{N}_{i-1}(\boldsymbol{q})-\overline{\boldsymbol{J}}_{i-1}(\boldsymbol{q})^{T} \overline{\boldsymbol{J}}_{i-1}(\boldsymbol{q})^{\boldsymbol{M}+, T}
\end{aligned}
$$

where

$$
\overline{\boldsymbol{J}}_{i}(\boldsymbol{q})^{\boldsymbol{M}+}:=\boldsymbol{M}(\boldsymbol{q})^{-1} \overline{\boldsymbol{J}}_{i}(\boldsymbol{q})^{T}\left(\overline{\boldsymbol{J}}_{i}(\boldsymbol{q}) \boldsymbol{M}(\boldsymbol{q})^{-1} \overline{\boldsymbol{J}}_{i}(\boldsymbol{q})^{T}\right)^{-1}
$$

is the dynamically consistent generalized inverse of $\overline{\boldsymbol{J}}_{i}(\boldsymbol{q})$ [1]. Since $\boldsymbol{J}(\boldsymbol{q})$ is nonsingular, the property $\operatorname{rank}\left(\overline{\boldsymbol{J}}_{i}(\boldsymbol{q})\right)=m_{i}$ holds for all levels $i=1 \ldots r$, as proven in Appendix A. Moreover, $\overline{\boldsymbol{J}}_{i}(\boldsymbol{q})^{\boldsymbol{M}+}$ exists because $\overline{\boldsymbol{J}}_{i}(\boldsymbol{q}) \boldsymbol{M}(\boldsymbol{q})^{-1} \overline{\boldsymbol{J}}_{i}(\boldsymbol{q})^{T}$ is invertible, as required by (12).

The hierarchy-consistent task-space velocity vector $v \in \mathbb{R}^{n}$ is defined as

$$
\boldsymbol{v}:=\overline{\boldsymbol{J}}(\boldsymbol{q}) \dot{\boldsymbol{q}}
$$

where $\overline{\boldsymbol{J}}(\boldsymbol{q})$ represents the stacked prioritized Jacobian matrix

$$
\overline{\boldsymbol{J}}(\boldsymbol{q}):=\left[\begin{array}{lll}
\overline{\boldsymbol{J}}_{1}^{T}(\boldsymbol{q}) & \ldots & \overline{\boldsymbol{J}}_{r}^{T}(\boldsymbol{q})
\end{array}\right]^{T} .
$$

The respective component of $\boldsymbol{v}$ on the $i$-th hierarchy level can be obtained by $\boldsymbol{v}_{i}:=\overline{\boldsymbol{J}}_{i}(\boldsymbol{q}) \dot{\boldsymbol{q}} \in \mathbb{R}^{m_{i}}$. Moreover, the inverse of $\overline{\boldsymbol{J}}(\boldsymbol{q})$ can be computed by [16] (see Appendix A)

$$
\overline{\boldsymbol{J}}(\boldsymbol{q})^{-1}=\left[\begin{array}{lll}
\overline{\boldsymbol{J}}_{1}(\boldsymbol{q})^{\boldsymbol{M}+} & \ldots & \overline{\boldsymbol{J}}_{r}(\boldsymbol{q})^{\boldsymbol{M}+}
\end{array}\right] .
$$

\section{E. Inertially Decoupled Dynamics}

Applying the coordinate transformation (13) to (1) yields the inertially decoupled task-space dynamics

$$
\boldsymbol{\Lambda}(\boldsymbol{q}) \dot{\boldsymbol{v}}+\boldsymbol{\mu}(\boldsymbol{q}, \dot{\boldsymbol{q}}) \boldsymbol{v}=\overline{\boldsymbol{J}}(\boldsymbol{q})^{-T}\left(\boldsymbol{\tau}+\boldsymbol{\tau}^{\mathrm{ext}}-\boldsymbol{g}(\boldsymbol{q})\right)
$$

In the following, dependencies on $\boldsymbol{q}, \dot{\boldsymbol{q}}$ are omitted for the sake of simplicity. The block diagonal inertia matrix is given by

$$
\boldsymbol{\Lambda}:=\overline{\boldsymbol{J}}^{-T} \boldsymbol{M} \overline{\boldsymbol{J}}^{-1}=\operatorname{diag}\left\{\boldsymbol{\Lambda}_{i}\right\}
$$

where $\boldsymbol{\Lambda}_{i}:=\left(\overline{\boldsymbol{J}}_{i} \boldsymbol{M}^{-1} \overline{\boldsymbol{J}}_{i}^{T}\right)^{-1} \in \mathbb{R}^{m_{i} \times m_{i}}$ denotes the subtask inertia matrix on the $i$-th hierarchy level [10]. The task-space Coriolis/centrifugal matrix can be derived as

$$
\boldsymbol{\mu}:=\left(\overline{\boldsymbol{J}}^{-T} \boldsymbol{C}-\boldsymbol{\Lambda} \dot{\overline{\boldsymbol{J}}}\right) \overline{\boldsymbol{J}}^{-1} \text {. }
$$

Although $\boldsymbol{\mu}$ still induces dynamic couplings across the task hierarchy due to its non-zero off-diagonal blocks, in general, it possesses a beneficial structure which has been exploited 
to facilitate passivity-based control design [14], [16], [17]. To elaborate, $\boldsymbol{\mu}$ can be expressed as

$$
\boldsymbol{\mu}=\overline{\boldsymbol{\mu}}+(\boldsymbol{\mu}-\overline{\boldsymbol{\mu}}),
$$

where $\overline{\boldsymbol{\mu}}:=\operatorname{diag}\left\{\boldsymbol{\mu}_{i, i}\right\}$ with $\boldsymbol{\mu}_{i, i} \in \mathbb{R}^{m_{i} \times m_{i}}$ being the diagonal block matrices of $\boldsymbol{\mu}$. On account of Assumption 2 and the block diagonal structure of $\boldsymbol{\Lambda}$, two properties can be shown: a) $\dot{\boldsymbol{\Lambda}}=\overline{\boldsymbol{\mu}}+\overline{\boldsymbol{\mu}}^{T}$ holds; b) $\boldsymbol{\mu}-\overline{\boldsymbol{\mu}}$ is skew-symmetric. The latter can be utilized to implement a power-conserving compensation of the cross-couplings $(\boldsymbol{\mu}-\overline{\boldsymbol{\mu}}) \boldsymbol{v}$, as the transmitted power is zero, i. e., $\boldsymbol{v}^{T}(\boldsymbol{\mu}-\overline{\boldsymbol{\mu}}) \boldsymbol{v}=\mathbf{0}$ [14]. Note that Assumption 1 together with the assumed nonsingularity of $\overline{\boldsymbol{J}}(\boldsymbol{q})$ guarantees the boundedness of $\boldsymbol{\Lambda}(\boldsymbol{q})$, and Assumption 3 further ensures that $\boldsymbol{\mu}(\boldsymbol{q}, \dot{\boldsymbol{q}})$ is bounded in $\boldsymbol{q}$.

\section{F. Relations Between Original and Hierarchy-Consistent Task-Space Velocities and Accelerations}

When designing a tracking control law based on the taskspace dynamics (16), a major challenge is to cope with the mismatch between the original and the hierarchy-consistent task-space quantities. More specifically, the left-hand side of (16) is represented with $\boldsymbol{v}$ and $\dot{v}$, whereas the control objective is to enforce the trajectory tracking w. r.t. $(\boldsymbol{x}, \dot{\boldsymbol{x}}, \ddot{\boldsymbol{x}})$. In this section the formalism from [17] is briefly recalled, which describes the relations between original and hierarchyconsistent task-space velocities and accelerations.

Assuming that $\boldsymbol{J}$ is nonsingular, the relation between $\boldsymbol{v}$ and $\dot{\boldsymbol{x}}$ can be written as

$$
\boldsymbol{v}=\boldsymbol{B} \dot{\boldsymbol{x}}
$$

where $\boldsymbol{B}:=\overline{\boldsymbol{J}} \boldsymbol{J}^{-1} \in \mathbb{R}^{n \times n}$ is a lower triangular block matrix. Its upper and diagonal blocks are given by

$$
\boldsymbol{B}_{i, j}=\mathbf{0} \text { for } j>i \text {, and } \boldsymbol{B}_{i, j}=\boldsymbol{I} \text { for } j=i,
$$

with $\boldsymbol{B}_{i, j} \in \mathbb{R}^{m_{i} \times m_{j}}$ denoting the $(i, j)$-block of $\boldsymbol{B}$. Note that the lower triangular blocks of $\boldsymbol{B}$ are not empty in general. Differentiating (20) with respect to time leads to

$$
\dot{\boldsymbol{v}}=\boldsymbol{B} \ddot{\boldsymbol{x}}+\dot{\boldsymbol{B}} \dot{\boldsymbol{x}}
$$

where $\dot{\boldsymbol{B}}$ (the time derivative of $\boldsymbol{B}$ ) is also a lower triangular block but it has zero blocks on its diagonal. The lower triangular structure of $\boldsymbol{B}$ and $\dot{\boldsymbol{B}}$ results from the dynamic consistency of the null-space projectors, which ensures that lower priority tasks cannot interfere with higher priority ones during the transient. However, top-down couplings can still occur at both velocity and acceleration levels via mappings of lower triangular parts of $\boldsymbol{B}$ and $\dot{\boldsymbol{B}}$.

\section{G. HPD+ Controller and Top-Down Disturbances}

The HPD+ control scheme [17] will be briefly reviewed and reformulated in a more compact way with the notations used here. It can be viewed as a direct extension of the hierarchical regulation controller [16] to trajectory tracking by introducing feedforward terms in a PD+ manner [34]. The general control law of the HPD+ can be expressed as

$$
\boldsymbol{\tau}=\boldsymbol{g}+\boldsymbol{\tau}_{\boldsymbol{\mu}}+\boldsymbol{\tau}_{\mathrm{pd}}+\boldsymbol{\tau}_{\mathrm{ff}}+\tau_{\mathrm{comp}}^{\mathrm{ext}},
$$

where

$$
\begin{aligned}
\boldsymbol{\tau}_{\mu} & :=\overline{\boldsymbol{J}}^{T}(\boldsymbol{\mu}-\overline{\boldsymbol{\mu}}) \boldsymbol{v}, \\
\boldsymbol{\tau}_{\mathrm{pd}} & :=\overline{\boldsymbol{J}}^{T}(-\boldsymbol{D} \dot{\tilde{\boldsymbol{x}}}-\boldsymbol{K} \tilde{\boldsymbol{x}}) \\
\boldsymbol{\tau}_{\mathrm{comp}}^{\mathrm{ext}} & :=\overline{\boldsymbol{J}}^{T}\left(\boldsymbol{I}-\boldsymbol{B}^{-T}\right) \boldsymbol{F}_{\dot{\boldsymbol{x}}}^{\mathrm{ext}}, \\
\boldsymbol{\tau}_{\mathrm{ff}} & :=\overline{\boldsymbol{J}}^{T}\left(\boldsymbol{\Lambda}\left(\boldsymbol{B} \ddot{\boldsymbol{x}}_{\mathrm{des}}+\dot{\boldsymbol{B}} \dot{\boldsymbol{x}}_{\mathrm{des}}\right)+\overline{\boldsymbol{\mu}} \boldsymbol{B} \dot{\boldsymbol{x}}_{\mathrm{des}}\right) .
\end{aligned}
$$

It contains the gravity compensation $\boldsymbol{g}$, the power-conserving feedback term $\tau_{\mu}$ to compensate for cross-couplings in $\boldsymbol{\mu}$, the proportional derivative (PD) control action $\tau_{\mathrm{pd}}$, the $o p$ tional feedback term $\tau_{\text {comp }}^{\text {ext }}$ to annihilate the bottom-up ${ }^{3}$ cross-couplings of $\boldsymbol{F}_{\dot{\boldsymbol{x}}}^{\text {ext }}$ in case of physical interaction, and the feedforward action $\boldsymbol{\tau}_{\mathrm{ff}}{ }^{4}$ The block diagonal matrices $\boldsymbol{K}:=\operatorname{diag}\left\{\boldsymbol{K}_{i}\right\}$ and $\boldsymbol{D}:=\operatorname{diag}\left\{\boldsymbol{D}_{i}\right\}$ describe the overall task-space stiffness and damping, respectively, with the symmetric and positive definite terms $\boldsymbol{K}_{i}, \boldsymbol{D}_{i} \in \mathbb{R}^{m_{i} \times m_{i}}$.

The control law (23) does not involve any inertia shaping, it requires no measurement/estimation of $\boldsymbol{\tau}^{\text {ext }}$ or $\boldsymbol{F}_{\dot{x}}^{\text {ext }}$ when $\tau_{\text {comp }}^{\text {ext }}$ is not applied, and it provides UAS in free motion $\left(\boldsymbol{\tau}^{\text {ext }}=\mathbf{0}\right)$. Nevertheless, except for the primary task $(i=1)$, the transient performance of the individual tasks is affected by internal top-down disturbances due to remaining crosscouplings. Applying (23) with (24)-(27) to (16) yields the closed-loop dynamics on the hierarchy level $i>1$

$$
\boldsymbol{\Lambda}_{i} \ddot{\tilde{\boldsymbol{x}}}_{i}+\left(\boldsymbol{\mu}_{i, i}+\boldsymbol{D}_{i}\right) \dot{\tilde{\boldsymbol{x}}}_{i}+\boldsymbol{K}_{i} \tilde{\boldsymbol{x}}_{i}-\boldsymbol{F}_{\dot{\boldsymbol{x}}_{i}}^{\mathrm{ext}}=\gamma_{i}
$$

with the internal top-down disturbances

$$
\boldsymbol{\gamma}_{i}:=-\boldsymbol{\Lambda}_{i} \sum_{j=1}^{i-1}\left(\boldsymbol{B}_{i, j} \ddot{\tilde{\boldsymbol{x}}}_{j}+\dot{\boldsymbol{B}}_{i, j} \dot{\tilde{\boldsymbol{x}}}_{j}\right)-\boldsymbol{\mu}_{i, i} \sum_{j=1}^{i-1} \boldsymbol{B}_{i, j} \dot{\tilde{\boldsymbol{x}}}_{j}
$$

When there remain task-space tracking errors on any higher priority level $j=1 \ldots i-1, \gamma_{i}$ will constantly disturb the desired closed-loop dynamics on the hierarchy level $i$, that is, the left-hand side of (28). In addition, the stable physical interaction can only be proved for the case of constant $\boldsymbol{F}_{\dot{\boldsymbol{x}}}^{\text {ext }}$, since any higher priority acceleration $\ddot{x}_{j}$ induced by some time-varying $\boldsymbol{F}_{\dot{\boldsymbol{x}}_{j}}^{\text {ext }}$ can generate disturbances along the closedloop trajectories on all lower levels $j+1 \ldots r$.

\section{Passive Decoupled Multi-Task Controller: DESIGN AND COMPARATIVE ANALYSIS}

\section{A. Controller Design}

Rather than to rely on the asymptotic convergence of the top-down disturbances [17], the proposed control law is designed to compensate for the transient top-down couplings in task space at velocity and acceleration level,

$$
\begin{aligned}
& \dot{\boldsymbol{w}}:=\boldsymbol{v}-\dot{\boldsymbol{x}}=(\boldsymbol{B}-\boldsymbol{I}) \dot{\boldsymbol{x}} \\
& \ddot{\boldsymbol{w}}:=\dot{\boldsymbol{v}}-\ddot{\boldsymbol{x}}=(\boldsymbol{B}-\boldsymbol{I}) \ddot{\boldsymbol{x}}+\dot{\boldsymbol{B}} \dot{\boldsymbol{x}} .
\end{aligned}
$$

\footnotetext{
${ }^{3}$ This is implied by the upper triangular structure of $\boldsymbol{B}^{-T}$.

${ }^{4}$ Since $\boldsymbol{\tau}_{\mathrm{ff}}$ also includes feedback of measured states, it is strictly speaking not a common feedforward action, but the desired trajectories are fed forward through it in a PD+ manner.
} 
This disturbance/coupling rejection is achieved by keeping the level-specific feedforward terms in (27) and compensating for the effects of $\dot{\boldsymbol{w}}$ and $\ddot{\boldsymbol{w}}$ through feedback actions, yielding

$$
\boldsymbol{\tau}_{\mathrm{ff}+\mathrm{comp}}:=\overline{\boldsymbol{J}}^{T}\left(\boldsymbol{\Lambda}\left(\ddot{\boldsymbol{x}}_{\mathrm{des}}+\ddot{\boldsymbol{w}}\right)+\overline{\boldsymbol{\mu}}\left(\dot{\boldsymbol{x}}_{\mathrm{des}}+\dot{\boldsymbol{w}}\right)\right) .
$$

However, the formulation of $\ddot{\boldsymbol{w}}$ in (31) requires the knowledge of $\ddot{\boldsymbol{x}}$ at each time instant, and it is thus not realizable due to the lack of causality. Therefore, $\ddot{\boldsymbol{x}}$ in (31) is replaced with the task-space reference acceleration

$$
\ddot{\boldsymbol{x}}_{\mathrm{ref}}:=\ddot{\boldsymbol{x}}_{\mathrm{des}}+\boldsymbol{\Lambda}^{-1}\left(\boldsymbol{F}_{\dot{\boldsymbol{x}}}^{\mathrm{ext}}-(\overline{\boldsymbol{\mu}}+\boldsymbol{D}) \dot{\tilde{\boldsymbol{x}}}-\boldsymbol{K} \tilde{\boldsymbol{x}}\right),
$$

which stems from the desired (decoupled) impedance behavior of the closed-loop system. In order to correctly reconstruct $\ddot{\boldsymbol{x}}_{\text {ref }}$ from (33) as an estimate of $\ddot{\boldsymbol{x}}$, one needs to annihilate the cross-couplings of external task-space forces/torques. As a result, $\tau_{\text {comp }}^{\text {ext }}$ becomes necessary, thereby requiring measurement/estimation of $\boldsymbol{\tau}^{\text {ext }}$ or $\boldsymbol{F}_{\dot{\boldsymbol{x}}}^{\text {ext }}$. It is worth mentioning that a similar formulation of $\ddot{\boldsymbol{x}}_{\text {ref }}$ has also been introduced in [31], but it is used for single-level regulation control, and the task-space interaction forces/torques are treated as external disturbances to be fully compensated.

Combining (24)-(26) with (30)-(32) into (23) yields

$$
\boldsymbol{\tau}=\boldsymbol{g}+\overline{\boldsymbol{J}}^{T} \boldsymbol{\mu} \boldsymbol{v}-\overline{\boldsymbol{J}}^{T} \boldsymbol{B}^{-T} \boldsymbol{F}_{\dot{\boldsymbol{x}}}^{\mathrm{ext}}+\overline{\boldsymbol{J}}^{T} \boldsymbol{\Lambda}\left(\boldsymbol{B} \ddot{\boldsymbol{x}}_{\mathrm{ref}}+\dot{\boldsymbol{B}} \dot{\boldsymbol{x}}\right) .
$$

One can observe that the task-space Coriolis/centrifugal forces $\boldsymbol{\mu} \boldsymbol{v}$ are first completely compensated for, and then their level-specific components $\overline{\boldsymbol{\mu}} \dot{\boldsymbol{x}}$ are recovered through (33). In [35] the authors suggested to fully (pre-)compensate for both Coriolis/centrifugal and gravitational terms in joint space. Besides, the pre-computation of the total matrix $\boldsymbol{\mu}$ is in fact not necessary as $\overline{\boldsymbol{\mu}}$ can be directly obtained through

$$
\boldsymbol{\mu}_{i, i}=\overline{\boldsymbol{J}}_{i}^{\boldsymbol{M +} T} \boldsymbol{C} \overline{\boldsymbol{J}}_{i}^{\boldsymbol{M +}}-\boldsymbol{\Lambda}_{i} \dot{\overline{\boldsymbol{J}}}_{i} \overline{\boldsymbol{J}}_{i}^{\boldsymbol{M +}} .
$$

Therefore, the joint-space compensation of Coriolis/centrifugal effects is adopted by applying (13) and (18) to (34):

$\boldsymbol{\tau}=\boldsymbol{g}+\boldsymbol{C} \dot{\boldsymbol{q}}-\overline{\boldsymbol{J}}^{T} \boldsymbol{B}^{-T} \boldsymbol{F}_{\dot{\boldsymbol{x}}}^{\mathrm{ext}}+\overline{\boldsymbol{J}}^{T} \boldsymbol{\Lambda}\left(\boldsymbol{B} \ddot{\boldsymbol{x}}_{\mathrm{ref}}+\dot{\boldsymbol{B}} \dot{\boldsymbol{x}}-\dot{\overline{\boldsymbol{J}}} \dot{\boldsymbol{q}}\right)$.

Substituting $\dot{\boldsymbol{B}}$ with

$$
\dot{\boldsymbol{B}}=\dot{\overline{\boldsymbol{J}}} \boldsymbol{J}^{-1}-\overline{\boldsymbol{J}} \boldsymbol{J}^{-1} \dot{\boldsymbol{J}} \boldsymbol{J}^{-1}=(\dot{\overline{\boldsymbol{J}}}-\boldsymbol{B} \dot{\boldsymbol{J}}) \boldsymbol{J}^{-1}
$$

in (36), one obtains the final control law

$$
\boldsymbol{\tau}=\boldsymbol{g}+\boldsymbol{C} \dot{\boldsymbol{q}}-\overline{\boldsymbol{J}}^{T} \boldsymbol{B}^{-T} \boldsymbol{F}_{\dot{\boldsymbol{x}}}^{\mathrm{ext}}+\overline{\boldsymbol{J}}^{T} \boldsymbol{\Lambda} \boldsymbol{B}\left(\ddot{\boldsymbol{x}}_{\mathrm{ref}}-\dot{\boldsymbol{J}} \dot{\boldsymbol{q}}\right)
$$

Remark 3: From a computational point of view, the straightforward implementation of (20) to compute $\boldsymbol{B}$ is not efficient, as it requires the inverse of $\boldsymbol{J}$ which costs $\mathcal{O}\left(n^{3}\right)$ of effort in real time. Since $\boldsymbol{B}^{-1}$ is lower triangular, the computation effort can be reduced to $\mathcal{O}\left(n^{2}\right)$ by solving $\boldsymbol{B}^{-1} \boldsymbol{z}=\ddot{\boldsymbol{x}}_{\text {ref }}-\dot{\boldsymbol{J}} \dot{\boldsymbol{q}}$ for $z \in \mathbb{R}^{n}$ [36, Sec.3.1.1]. Moreover, the $(i, j)$-block of $\boldsymbol{B}^{-1}$ for $i>j$ can be directly computed as $\boldsymbol{J}_{i} \overline{\boldsymbol{J}}_{j}^{M+}$.

The control law (38) together with (33) leads to the reference behavior $\ddot{\boldsymbol{x}}=\ddot{\boldsymbol{x}}_{\text {ref }}$. Consequently, it results in the desired closed-loop equations of motion

$$
\boldsymbol{\Lambda} \ddot{\tilde{\boldsymbol{x}}}+(\overline{\boldsymbol{\mu}}+\boldsymbol{D}) \dot{\tilde{\boldsymbol{x}}}+\boldsymbol{K} \tilde{\boldsymbol{x}}=\boldsymbol{F}_{\dot{\boldsymbol{x}}}^{\mathrm{ext}},
$$

where the internal top-down disturbances (29) are now fully canceled on all levels $i=2 \ldots r$. Although (39) describes a hierarchically decoupled system, note that there remain state dependencies in $\boldsymbol{\Lambda}_{i}(\boldsymbol{q})$ and $\boldsymbol{\mu}_{i, i}(\boldsymbol{q}, \dot{\boldsymbol{q}})$ across the task hierarchy.

\section{B. Comparison with Feedback Linearization}

The control scheme (38) does not restrict the concrete objectives of the reference accelerations. As a result, it can also be used to implement the multi-objective controllers based on feedback linearization. In general, the impedance-based reference acceleration for the feedback linearization can be written as

$$
\ddot{\boldsymbol{x}}_{\mathrm{ref}, \mathrm{fl}}:=\ddot{\boldsymbol{x}}_{\mathrm{des}}+\boldsymbol{\Lambda}_{\mathrm{des}}^{-1}\left(\boldsymbol{F}_{\dot{\boldsymbol{x}}}^{\mathrm{ext}}-\boldsymbol{D} \dot{\tilde{\boldsymbol{x}}}-\boldsymbol{K} \tilde{\boldsymbol{x}}\right)
$$

with $\boldsymbol{\Lambda}_{\text {des }}:=\operatorname{diag}\left\{\boldsymbol{\Lambda}_{i, \text { des }}\right\}$. Herein, $\boldsymbol{\Lambda}_{i, \text { des }} \in \mathbb{R}^{m_{i} \times m_{i}}$ describes the constant, desired subtask inertia which is often chosen to be the identity matrix. Note that (40) does not involve the reconstruction of the Coriolis/centrifugal term $\overline{\boldsymbol{\mu}} \dot{\tilde{x}}$, in contrast to the proposed control law.

1) Distortion of Feedback Gains: Applying (40) in (38) and recalling $\boldsymbol{B}_{i i}=\boldsymbol{I}$ from (21), one can observe that before mapped onto the joint torque space via $\overline{\boldsymbol{J}}_{i}^{T}$, the task-space PD control action on the hierarchy level $i$, that is,

$$
\boldsymbol{F}_{i, \mathrm{pd}}:=-\boldsymbol{D}_{i} \dot{\tilde{\boldsymbol{x}}}_{i}-\boldsymbol{K}_{i} \tilde{\boldsymbol{x}}_{i}
$$

is pre-multiplied by $\boldsymbol{\Lambda}_{i} \boldsymbol{\Lambda}_{i \text {,des }}^{-1}$ in feedback linearization. This configuration-dependent distortion effect can largely degrade the tracking performance when $\boldsymbol{\Lambda}_{i, \text { des }}$ is not well-tuned. Moreover, it can also increase the risk of exceeding actuator limits due to unrealistic inertial responses required by $\boldsymbol{\Lambda}_{i, \mathrm{des}}$. On the other hand, using the natural task-space inertia (i.e., $\boldsymbol{\Lambda}_{i \text {, des }}=\boldsymbol{\Lambda}_{i}$ ) in $\ddot{\boldsymbol{x}}_{i \text {,ref }}$, as in (33), eliminates this effect. Notice that for one-dimensional tasks, $\boldsymbol{\Lambda}_{i} \boldsymbol{\Lambda}_{i, \text { des }}^{-1}$ is simplified to a scalar factor that either amplifies or attenuates $\boldsymbol{F}_{i, \mathrm{pd}}$ directly.

In order to evaluate this distortion effect across the task hierarchy, the analytical expression of the lower triangular parts of $\boldsymbol{B}$ is derived as (see Appendix B for details)

$$
\boldsymbol{B}_{i, j}=-\boldsymbol{J}_{i} \prod_{k=j+1}^{i-1} \boldsymbol{\Phi}_{k} \overline{\boldsymbol{J}}_{j}^{M+}, \forall i>j
$$

where $\boldsymbol{\Phi}_{k}:=\boldsymbol{I}-\overline{\boldsymbol{J}}_{k}^{\boldsymbol{M}+} \boldsymbol{J}_{k}$. Then, using the natural task inertia on a higher level $j<i$ (i.e., $\boldsymbol{\Lambda}_{j \text {,des }}=\boldsymbol{\Lambda}_{j}$ ) leads to

$$
\begin{aligned}
\boldsymbol{\Lambda}_{i} \boldsymbol{B}_{i, j} \boldsymbol{\Lambda}_{j, \mathrm{des}}^{-1} & =-\boldsymbol{\Lambda}_{i} \boldsymbol{J}_{i} \prod_{k=j+1}^{i-1} \boldsymbol{\Phi}_{k} \boldsymbol{M}^{-1} \overline{\boldsymbol{J}}_{j}^{T} \boldsymbol{\Lambda}_{j} \boldsymbol{\Lambda}_{j}^{-1} \\
& =-\boldsymbol{\Lambda}_{i} \boldsymbol{J}_{i} \prod_{k=j+1}^{i-1} \boldsymbol{\Phi}_{k} \boldsymbol{M}^{-1} \overline{\boldsymbol{J}}_{j}^{T}
\end{aligned}
$$

Thus, the proposed controller does not induce the distortion effect of $\boldsymbol{\Lambda}_{j} \boldsymbol{\Lambda}_{j \text {,des }}^{-1}$ on all lower levels $i=j+1 \ldots r$, which will otherwise arise in the case of feedback linearization. 
2) Error Propagation of External Force Feedback: As both approaches require the feedback of external forces/torques, it is of special interest to analyze the error propagation of such feedback into their closed-loop dynamics. Let $\hat{\boldsymbol{F}}_{\dot{\boldsymbol{x}}}^{\text {ext }}$ denote the generalized task-space external forces obtained by estimation or measurement, and $\tilde{\boldsymbol{F}}_{\dot{\boldsymbol{x}}}^{\text {ext }}:=\boldsymbol{F}_{\dot{\boldsymbol{x}}}^{\mathrm{ext}}-\hat{\boldsymbol{F}}_{\dot{\boldsymbol{x}}}^{\text {ext }}$ the corresponding estimation/measurement errors. Replacing $\boldsymbol{F}_{\dot{\boldsymbol{x}}}^{\text {ext }}$ with $\hat{\boldsymbol{F}}_{\dot{\boldsymbol{x}}}^{\text {ext }}$ in the control law (38) yields

$$
\boldsymbol{\tau}=\boldsymbol{g}+\boldsymbol{C} \dot{\boldsymbol{q}}-\overline{\boldsymbol{J}}^{T} \boldsymbol{B}^{-T} \hat{\boldsymbol{F}}_{\dot{\boldsymbol{x}}}^{\mathrm{ext}}+\overline{\boldsymbol{J}}^{T} \boldsymbol{\Lambda} \boldsymbol{B}\left(\ddot{\hat{\boldsymbol{x}}}_{\mathrm{ref}}-\dot{\boldsymbol{J}} \dot{\boldsymbol{q}}\right)
$$

where $\ddot{\hat{\boldsymbol{x}}}_{\mathrm{ref}}:=\ddot{\boldsymbol{x}}_{\mathrm{ref}}-\boldsymbol{\Lambda}^{-1} \tilde{\boldsymbol{F}}_{\dot{\boldsymbol{x}}}^{\text {ext }}$ describes the task-space reference acceleration perturbed by $\tilde{\boldsymbol{F}}_{\dot{\boldsymbol{x}}}^{\text {ext }}$. As a result, the closedloop dynamics becomes

$$
\begin{aligned}
\ddot{\boldsymbol{x}} & =\ddot{\hat{\boldsymbol{x}}}_{\mathrm{ref}}+\boldsymbol{B}^{-1} \boldsymbol{\Lambda}^{-1} \boldsymbol{B}^{-T} \tilde{\boldsymbol{F}}_{\dot{\boldsymbol{x}}}^{\mathrm{ext}} \\
& =\ddot{\boldsymbol{x}}_{\mathrm{ref}}+\left(\boldsymbol{B}^{-1} \boldsymbol{\Lambda}^{-1} \boldsymbol{B}^{-T}-\boldsymbol{\Lambda}^{-1}\right) \tilde{\boldsymbol{F}}_{\dot{\boldsymbol{x}}}^{\mathrm{ext}}
\end{aligned}
$$

Similarly, the perturbed closed-loop dynamics in case of the feedback linearization is given by

$$
\ddot{\boldsymbol{x}}=\ddot{\boldsymbol{x}}_{\mathrm{ref}, \mathrm{fl}}+\left(\boldsymbol{B}^{-1} \boldsymbol{\Lambda}^{-1} \boldsymbol{B}^{-T}-\boldsymbol{\Lambda}_{\mathrm{des}}^{-1}\right) \tilde{\boldsymbol{F}}_{\dot{\boldsymbol{x}}}^{\mathrm{ext}} .
$$

Clearly, since the matrix $\boldsymbol{B}^{-1} \boldsymbol{\Lambda}^{-1} \boldsymbol{B}^{-T}$ is, in general, fully occupied, both (45) and (46) can no longer achieve decoupled closed-loop dynamics when $\tilde{\boldsymbol{F}}_{\dot{\boldsymbol{x}}}^{\text {ext }}$ is non-zero. However, the proposed approach is less sensitive against $\tilde{\boldsymbol{F}}_{\dot{\boldsymbol{x}}}^{\text {ext }}$ as compared to the feedback linearization, at least for an interesting special case. This is when $\tilde{\boldsymbol{F}}_{\dot{\boldsymbol{x}}}^{\text {ext }}$ only has non-zero components corresponding to the first priority level, i. e., $\tilde{\boldsymbol{F}}_{\dot{\boldsymbol{x}}_{1}}^{\text {ext }} \neq \mathbf{0}$ and $\tilde{\boldsymbol{F}}_{\dot{\boldsymbol{x}}_{i}}^{\text {ext }}=\mathbf{0}$ for all levels $i=2 \ldots r$. Because the $(1,1)$-block of $\boldsymbol{B}^{-1} \boldsymbol{\Lambda}^{-1} \boldsymbol{B}^{-T}$ is equal to $\boldsymbol{\Lambda}_{1}^{-1}$, the performance of the main task with the proposed approach will not be influenced by $\tilde{\boldsymbol{F}}_{\dot{\boldsymbol{x}}}^{\text {ext }}$, whereas the performance with the feedback linearization will degenerate due to $\boldsymbol{\Lambda}_{1, \text { des }}^{-1} \neq \boldsymbol{\Lambda}_{1}^{-1}$. For a more general case, the $(i, i)$-block of $\boldsymbol{B}^{-1} \boldsymbol{\Lambda}^{-1} \boldsymbol{B}^{-T}$ is equivalent to the inverse of the operational-space kinetic energy matrix given by [3]

$$
\boldsymbol{\Lambda}_{i, \mathrm{kin}}:=\left(\boldsymbol{J}_{i} \boldsymbol{M}^{-1} \boldsymbol{J}_{i}^{T}\right)^{-1} \in \mathbb{R}^{m_{i} \times m_{i}} .
$$

Note that $\boldsymbol{\Lambda}_{i, \mathrm{kin}}$ is different from $\boldsymbol{\Lambda}_{i}$ except for the first priority level, since it is computed with the original Jacobian matrices which are not hierarchy-consistent on lower levels.

\section{Stability and Passivity Analysis}

In this section the stability properties of the equilibrium

$$
\tilde{\boldsymbol{x}}_{i}=\dot{\tilde{\boldsymbol{x}}}_{i}=\mathbf{0}, \quad \forall i=1 \ldots r
$$

of the overall closed-loop system in case of free motion and under bounded interaction forces are analyzed. It should be noted that due to the assumed restriction to nonsingular task space, the initial conditions of the results cannot include the entire state space $\boldsymbol{q}, \dot{\boldsymbol{q}} \in \mathbb{R}^{n}$. Thus, the stability properties are only valid in the local sense, which is a common limitation of stability analyses for task-space controllers [7], [17], [19]. In addition to stability, the passivity of the closed-loop system w.r. t. the input-output-pair $\left(\boldsymbol{F}_{\dot{\boldsymbol{x}}}^{\text {ext }}, \dot{\tilde{\boldsymbol{x}}}\right)$ is also clarified.

\section{A. Scaling Metrics for Physically Consistent Norms}

In general, the dimensionless Euclidean norm is not suitable for quantitative evaluation of a vector/covector with components of different physical units of measurement [37], [38]. For example, the total velocity error vector $\dot{\tilde{\boldsymbol{x}}}$ may contain linear and angular components which are measured in $\mathrm{m} / \mathrm{s}$ and $\mathrm{rad} / \mathrm{s}$, respectively. The Euclidean norm $\|\dot{\tilde{\boldsymbol{x}}}\|$ is physically meaningless as its physical unit cannot be determined. Therefore, some scaling metrics inspired by [39] have to be introduced in order to properly define a set of physically consistent norms used for the following analysis.

One can select a diagonal, positive definite matrix $\boldsymbol{W}_{x}:=\operatorname{diag}\left\{w_{x, i}\right\}$ with $i=1 \ldots n$ as a metric tensor on the vector space of $\boldsymbol{x}$, such that the inner product

$$
\boldsymbol{x}^{T} \boldsymbol{W}_{x} \boldsymbol{x}=\sum_{i=1}^{n} w_{x, i} x_{i}^{2}
$$

w.r.t. the metric $\boldsymbol{W}_{x}$ has the unified unit of $\mathbf{J}$ (Joule), and the terms in the sum have comparable values. Note that $w_{x, i}$ is required to have an appropriate derived unit with respect to the unit of measurement of $x_{i}$, that is, $\left[w_{x, i}\right]=\mathrm{J} /\left[x_{i}\right]^{2}$. By an abuse of notation $[\cdot]$ denotes, from now on, the physical unit of the respective parameter or variable. Similarly, a diagonal positive definite matrix $\boldsymbol{W}_{\dot{x}}:=\operatorname{diag}\left\{w_{\dot{x}, i}\right\}$ can be used as a scaling metric for $\dot{\boldsymbol{x}}$ by enforcing $\left[w_{\dot{x}, i}\right]=\mathbf{J} /\left[\dot{x}_{i}\right]^{2}=\left[w_{x, i}\right] \cdot \mathrm{s}^{2}$. Moreover, since the scalar product $\dot{\boldsymbol{x}}^{T} \boldsymbol{F}_{\dot{\boldsymbol{x}}}^{\text {ext }}$ is supposed to have the fixed unit of $\mathrm{J} / \mathrm{s}$ or $\mathrm{W}$ (Watt), respectively, $\boldsymbol{W}_{\dot{x}}^{-1}$ can serve as a valid metric for $\boldsymbol{F}_{\dot{\boldsymbol{x}}}^{\text {ext }}$. This allows the unit of the inner product $\boldsymbol{F}_{\dot{\boldsymbol{x}}}^{\mathrm{ext}, T} \boldsymbol{W}_{\dot{x}}^{-1} \boldsymbol{F}_{\dot{\boldsymbol{x}}}^{\mathrm{ext}}$ to be determined as $\mathrm{J} / \mathrm{s}^{2}$. Summarized, a set of physically consistent norms induced by the associated scaled inner products can be defined as

$$
\begin{aligned}
&\|\tilde{\boldsymbol{x}}\|_{\boldsymbol{W}_{x}}:=\sqrt{\tilde{\boldsymbol{x}}^{T} \boldsymbol{W}_{x} \tilde{\boldsymbol{x}}}, \\
&\|\dot{\tilde{\boldsymbol{x}}}\|_{\boldsymbol{W}_{\dot{x}}}:=\sqrt{\dot{\tilde{\boldsymbol{x}}} \boldsymbol{W}_{\dot{x}} \dot{\tilde{\boldsymbol{x}}}}, \\
&\left\|\boldsymbol{F}_{\dot{\boldsymbol{x}}}^{\mathrm{ext}}\right\|_{\boldsymbol{W}_{\dot{x}}^{-1}}:=\sqrt{\boldsymbol{F}_{\dot{\boldsymbol{x}}}^{\mathrm{ext}, T} \boldsymbol{W}_{\dot{x}}^{-1} \boldsymbol{F}_{\dot{\boldsymbol{x}}}^{\mathrm{ext}}},
\end{aligned}
$$

with $\left[\|\tilde{\boldsymbol{x}}\|_{\boldsymbol{W}_{x}}^{2}\right]=\mathrm{J},\left[\|\dot{\tilde{\boldsymbol{x}}}\|_{\boldsymbol{W}_{\dot{x}}}^{2}\right]=\mathrm{J}$, and $\left[\left\|\boldsymbol{F}_{\dot{\boldsymbol{x}}}^{\mathrm{ext}}\right\|_{\boldsymbol{W}_{\dot{x}}^{-1}}^{2}\right]=\mathrm{J} / \mathrm{s}^{2}$.

\section{B. Scaled Error Variables and System Matrices}

Based on the norms (49) and (50), the vector of scaled error variables is defined as

$$
\boldsymbol{y}:=\left[\begin{array}{cc}
\boldsymbol{W}_{x}^{1 / 2} & \mathbf{0} \\
\mathbf{0} & \boldsymbol{W}_{\dot{x}}^{1 / 2}
\end{array}\right]\left[\begin{array}{c}
\tilde{\boldsymbol{x}} \\
\dot{\tilde{\boldsymbol{x}}}
\end{array}\right] \in \mathbb{R}^{2 n},
$$

where $\boldsymbol{W}_{x}^{1 / 2}$ and $\boldsymbol{W}_{\dot{x}}^{1 / 2}$ represent the principal square roots of the respective scaling matrices. Because the components of $\boldsymbol{y}$ are well-defined with the same unit, the Euclidean norm $\|\boldsymbol{y}\|=\sqrt{\|\tilde{\boldsymbol{x}}\|_{\boldsymbol{W}_{x}}^{2}+\|\dot{\tilde{\boldsymbol{x}}}\|_{\boldsymbol{W}_{\dot{x}}}^{2}}$ is physically consistent, and $\left[\boldsymbol{y}^{T} \boldsymbol{y}\right]=\mathrm{J}$. Also, note that (48) is equivalent to $\boldsymbol{y}=\mathbf{0}$ as the linear mapping in (52) is invertible. Additionally, the following scaled system matrices are needed for the stability analysis:

$$
\boldsymbol{\Lambda}_{\dot{x} \dot{x}}, \boldsymbol{\Lambda}_{x \dot{x}}, \boldsymbol{D}_{\dot{x} \dot{x}}, \boldsymbol{D}_{x \dot{x}}, \overline{\boldsymbol{\mu}}_{\dot{x} \dot{x}}, \overline{\boldsymbol{\mu}}_{x \dot{x}}, \boldsymbol{K}_{x x} .
$$

Herein, the first lower index indicates the left multiplication of the respective inverse square root and the second indicates 
the right multiplication, e.g., $\boldsymbol{\Lambda}_{x \dot{x}}:=\boldsymbol{W}_{x}^{-1 / 2} \boldsymbol{\Lambda} \boldsymbol{W}_{\dot{x}}^{-1 / 2}$. It can be easily verified that the scaled system matrices are either dimensionless or have unified units. This justifies the usage of their eigenvalues and induced Euclidean norms for the following analysis in terms of physical consistency. Furthermore, notice that the properties $\boldsymbol{\Lambda}_{\dot{x} \dot{x}}, \boldsymbol{D}_{\dot{x} \dot{x}}, \boldsymbol{K}_{x x} \succ 0$, $\dot{\boldsymbol{\Lambda}}_{\dot{x} \dot{x}}=\overline{\boldsymbol{\mu}}_{\dot{x} \dot{x}}+\overline{\boldsymbol{\mu}}_{\dot{x} \dot{x}}^{T}$, and $\dot{\boldsymbol{\Lambda}}_{x \dot{x}}=\overline{\boldsymbol{\mu}}_{x \dot{x}}+\overline{\boldsymbol{\mu}}_{x \dot{x}}^{T}$ are invariant with respect to the selected scaling metrics.

\section{Proof of Uniform Exponential Stability}

The following proposition shows that UES of the equilibrium can be achieved by the proposed controller, despite that nonlinearity of the system is partially retained in order to preserve the natural inertial properties.

Proposition 1: Consider the system (1) with the control law (38). Suppose that Assumptions 1-5 hold. Then, the equilibrium $\boldsymbol{y}=\mathbf{0}$ is uniformly exponentially stable for the case of free motion $\left(\tau^{\mathrm{ext}}=\mathbf{0}\right)$.

Proof: The proof of Proposition 1 is conducted by constructing a strict Lyapunov function with skewed level sets. As an intermediate step, uniform stability (US) of the equilibrium is first established in order to verify some boundedness conditions. For this purpose, an energy-like Lyapunov function can be intuitively chosen as

$$
V(t, \boldsymbol{y}):=\frac{1}{2} \boldsymbol{y}^{T} \boldsymbol{P}^{\mathrm{ene}} \boldsymbol{y}, \quad \boldsymbol{P}^{\mathrm{ene}}:=\left[\begin{array}{cc}
\boldsymbol{K}_{x x} & \mathbf{0} \\
\mathbf{0} & \boldsymbol{\Lambda}_{\dot{x} \dot{x}}
\end{array}\right] .
$$

It consists of artificial kinetic and potential energies of the error dynamics, i.e. $V(t, \boldsymbol{y})=\frac{1}{2} \dot{\tilde{\boldsymbol{x}}}^{T} \boldsymbol{\Lambda} \dot{\tilde{\boldsymbol{x}}}+\frac{1}{2} \tilde{\boldsymbol{x}}^{T} \boldsymbol{K} \tilde{\boldsymbol{x}}$.

Since $\boldsymbol{P}^{\text {ene }}$ is bounded and $\boldsymbol{P}^{\text {ene }} \succ 0, V(t, \boldsymbol{y})$ is decrescent and positive definite in $\boldsymbol{y}$ [40], [41]. Furthermore, by using $\dot{\boldsymbol{\Lambda}}_{\dot{x} \dot{x}}=\overline{\boldsymbol{\mu}}_{\dot{x} \dot{x}}+\overline{\boldsymbol{\mu}}_{\dot{x} \dot{x}}^{T}$, the time derivative of $V(t, \boldsymbol{y})$ along the solution of (39) in free motion can be obtained as ${ }^{5}$

$$
\dot{V}(t, \boldsymbol{y})=-\boldsymbol{y}^{T}\left[\begin{array}{cc}
\mathbf{0} & \mathbf{0} \\
\mathbf{0} & \boldsymbol{D}_{\dot{x} \dot{x}}
\end{array}\right] \boldsymbol{y} .
$$

In view of $\boldsymbol{D}_{\dot{x} \dot{x}} \succ 0, \dot{V}(t, \boldsymbol{y})$ is negative semi-definite in $\boldsymbol{y}$. Thus, based on Lyapunov's direct method [40, Theorem 5.3.14], US of the equilibrium $\boldsymbol{y}=\mathbf{0}$ can be concluded. Consequently, the tracking errors $\tilde{\boldsymbol{x}}$ and $\dot{\tilde{\boldsymbol{x}}}$ are bounded. This further implies the boundedness of $\boldsymbol{x}$ and $\dot{x}$ since the reference trajectories described by $\boldsymbol{x}_{\mathrm{des}}$ and $\dot{\boldsymbol{x}}_{\mathrm{des}}$ are bounded by definition. Moreover, as $\boldsymbol{J}$ is assumed to be nonsingular, $\dot{\boldsymbol{q}}$ is bounded due to $\dot{\boldsymbol{q}}=\boldsymbol{J}^{-1} \dot{\boldsymbol{x}}$, and in turn, $\overline{\boldsymbol{\mu}}(\boldsymbol{q}, \dot{\boldsymbol{q}})$ is bounded as well.

Next, the original level sets of $V(t, \boldsymbol{y})$ are skewed by introducing a cross-term $\epsilon \tilde{\boldsymbol{x}}^{T} \boldsymbol{\Lambda} \dot{\tilde{\boldsymbol{x}}}=\epsilon \tilde{\boldsymbol{x}}^{T} \boldsymbol{W}_{x}^{1 / 2} \boldsymbol{\Lambda}_{x \dot{x}} \boldsymbol{W}_{\dot{x}}^{1 / 2} \dot{\tilde{\boldsymbol{x}}}$ with $\epsilon>0$ and $[\epsilon]=1 / \mathrm{s}$ [23], leading to ${ }^{6}$

$$
V_{\epsilon}(t, \boldsymbol{y}):=\frac{1}{2} \boldsymbol{y}^{T} \boldsymbol{P}_{\epsilon}^{\mathrm{ene}} \boldsymbol{y}, \quad \boldsymbol{P}_{\epsilon}^{\mathrm{ene}}:=\left[\begin{array}{cc}
\boldsymbol{K}_{x x} & \epsilon \boldsymbol{\Lambda}_{x \dot{x}} \\
\epsilon \boldsymbol{\Lambda}_{x \dot{x}}^{T} & \boldsymbol{\Lambda}_{\dot{x} \dot{x}}
\end{array}\right] .
$$

In [17], [20], it has been proven that for sufficiently small and positive $\epsilon, V_{\epsilon}(t, \boldsymbol{y})$ is a valid strict Lyapunov function.

\footnotetext{
${ }^{5}$ Note that the time dependency in $\dot{V}(t, \boldsymbol{y})$ is due to possible configuration dependencies in the damping matrices $\boldsymbol{D}_{i}(\boldsymbol{q})$ for $i=1 \ldots r$.

${ }^{6}$ It can be shown that $\epsilon \boldsymbol{\Lambda}_{x \dot{x}}$ is dimensionless and so is $\boldsymbol{P}_{\epsilon}^{\text {ene }}$.
}

However, to the best of our knowledge, the determination of a concrete range for $\epsilon$ has only been addressed in joint-space control [21], [22], [24], but one has to use auxiliary functions in the cross-term which may not be physical intuitive.

Notice that $\epsilon$ is not used as an additional design parameter, but only the existence of $\epsilon$ within a proper range is needed to conclude the strictness of $V_{\epsilon}(t, \boldsymbol{y})$.

In the following, such a proper range for $\epsilon$ is derived without using any auxiliary functions in the cross-term. Under the condition $\boldsymbol{K}_{x x} \succ 0$, one can state that $\boldsymbol{P}_{\epsilon}^{\text {ene }} \succ 0$ iff the Schur complement [42, A.5.5]

$$
\boldsymbol{S}_{\epsilon}^{\mathrm{ene}}:=\boldsymbol{\Lambda}_{\dot{x} \dot{x}}-\epsilon^{2} \boldsymbol{\Lambda}_{x \dot{x}}^{T} \boldsymbol{K}_{x x}^{-1} \boldsymbol{\Lambda}_{x \dot{x}}
$$

of $\boldsymbol{K}_{x x}$ w.r.t. $\boldsymbol{P}_{\epsilon}^{\text {ene }}$ is positive definite. Based on the eigenvalue sensitivity theorem of symmetric matrices [36, Corollary 8.1.6], see also Appendix C, the eigenvalues of $\boldsymbol{S}_{\epsilon}^{\text {ene }}$ do not move by more than the induced Euclidean norm of the perturbation matrix $\epsilon^{2} \boldsymbol{\Lambda}_{x \dot{x}}^{T} \boldsymbol{K}_{x x}^{-1} \boldsymbol{\Lambda}_{x \dot{x}}$ w. r. t. $\boldsymbol{\Lambda}_{\dot{x} \dot{x}}$. Thus, $\lambda_{\mathrm{m}}\left(\boldsymbol{S}_{\epsilon}^{\mathrm{ene}}\right)$ is lower bounded by ${ }^{7}$

$$
\begin{aligned}
\lambda_{\mathrm{m}}\left(\boldsymbol{S}_{\epsilon}^{\mathrm{ene}}\right) & \geq \lambda_{\mathrm{m}}\left(\boldsymbol{\Lambda}_{\dot{x} \dot{x}}\right)-\epsilon^{2}\left\|\boldsymbol{\Lambda}_{x \dot{x}}^{T} \boldsymbol{K}_{x x}^{-1} \boldsymbol{\Lambda}_{x \dot{x}}\right\| \\
& \geq \lambda_{\mathrm{m}}\left(\boldsymbol{\Lambda}_{\dot{x} \dot{x}}\right)-\epsilon^{2} \frac{\left\|\boldsymbol{\Lambda}_{x \dot{x}}\right\|^{2}}{\lambda_{\mathrm{m}}\left(\boldsymbol{K}_{x x}\right)} .
\end{aligned}
$$

It follows that for any $\epsilon$ satisfying

$$
0<\epsilon<\frac{\sqrt{\lambda_{\mathrm{m}}\left(\boldsymbol{\Lambda}_{\dot{x} \dot{x}}\right) \lambda_{\mathrm{m}}\left(\boldsymbol{K}_{x x}\right)}}{\left\|\boldsymbol{\Lambda}_{x \dot{x}}\right\|}:=\epsilon^{\text {ene }},
$$

$\boldsymbol{S}_{\epsilon}^{\text {ene }}$ and $\boldsymbol{P}_{\epsilon}^{\text {ene }}$ are positive definite. Note that the upper bound $\epsilon^{\epsilon}$ ene always exists, as $\boldsymbol{\Lambda}_{\dot{x} \dot{x}}, \boldsymbol{K}_{x x} \succ 0$ and $\boldsymbol{\Lambda}_{x \dot{x}} \neq \mathbf{0}$. The time derivative of $V_{\epsilon}(t, \boldsymbol{y})$ is given by

$$
\dot{V}_{\epsilon}(t, \boldsymbol{y})=-\boldsymbol{y}^{T} \boldsymbol{P}_{\epsilon}^{\text {pow }} \boldsymbol{y}
$$

with

$$
\boldsymbol{P}_{\epsilon}^{\text {pow }}:=\left[\begin{array}{cc}
\epsilon \boldsymbol{K}_{x x} & \frac{1}{2} \epsilon\left(\boldsymbol{D}_{x \dot{x}}-\overline{\boldsymbol{\mu}}_{x \dot{x}}^{T}\right) \\
\frac{1}{2} \epsilon\left(\boldsymbol{D}_{x \dot{x}}-\overline{\boldsymbol{\mu}}_{x \dot{x}}^{T}\right)^{T} & \boldsymbol{D}_{\dot{x} \dot{x}}-\epsilon \boldsymbol{\Lambda}_{\dot{x} \dot{x}}
\end{array}\right]
$$

and $\left[\boldsymbol{P}_{\epsilon}^{\text {pow }}\right]=1 /$ s. Likewise, since $\epsilon \boldsymbol{K}_{x x} \succ 0$ holds by definition, $\boldsymbol{P}_{\epsilon}^{\text {pow }} \succ 0$ is satisfied iff the Schur complement

$\boldsymbol{S}_{\epsilon}^{\text {pow }}:=\boldsymbol{D}_{\dot{x} \dot{x}}-\epsilon\left(\boldsymbol{\Lambda}_{\dot{x} \dot{x}}+\frac{1}{4}\left(\boldsymbol{D}_{x \dot{x}}-\overline{\boldsymbol{\mu}}_{x \dot{x}}^{T}\right)^{T} \boldsymbol{K}_{x x}^{-1}\left(\boldsymbol{D}_{x \dot{x}}-\overline{\boldsymbol{\mu}}_{x \dot{x}}^{T}\right)\right)$

of $\epsilon \boldsymbol{K}_{x x}$ in $\boldsymbol{P}_{\epsilon}^{\text {pow }}$ is positive definite. Similar to (57), based on the fact that $\boldsymbol{S}_{\epsilon}^{\text {pow }}$ and $\boldsymbol{D}_{\dot{x} \dot{x}}$ are symmetric, $\lambda_{\mathrm{m}}\left(\boldsymbol{S}_{\epsilon}^{\text {pow }}\right)$ is lower bounded by

$$
\begin{aligned}
\lambda_{\mathrm{m}}\left(\boldsymbol{S}_{\epsilon}^{\mathrm{pow}}\right) \geq & \lambda_{\mathrm{m}}\left(\boldsymbol{D}_{\dot{x} \dot{x}}\right)-\epsilon\left\|\boldsymbol{\Lambda}_{\dot{x} \dot{x}}\right\| \\
& -\frac{\epsilon}{4}\left\|\left(\boldsymbol{D}_{x \dot{x}}-\overline{\boldsymbol{\mu}}_{x \dot{x}}^{T}\right)^{T} \boldsymbol{K}_{x x}^{-1}\left(\boldsymbol{D}_{x \dot{x}}-\overline{\boldsymbol{\mu}}_{x \dot{x}}^{T}\right)\right\| \\
\geq & \lambda_{\mathrm{m}}\left(\boldsymbol{D}_{\dot{x} \dot{x}}\right)-\epsilon \lambda_{\mathrm{M}}\left(\boldsymbol{\Lambda}_{\dot{x} \dot{x}}\right)-\frac{1}{4} \epsilon \frac{\left\|\boldsymbol{D}_{x \dot{x}}-\overline{\boldsymbol{\mu}}_{x \dot{x}}^{T}\right\|^{2}}{\lambda_{\mathrm{m}}\left(\boldsymbol{K}_{x x}\right)} .
\end{aligned}
$$

It follows that for any $\epsilon$ satisfying

$$
0<\epsilon<\frac{\lambda_{\mathrm{m}}\left(\boldsymbol{K}_{x x}\right) \lambda_{\mathrm{m}}\left(\boldsymbol{D}_{\dot{x} \dot{x}}\right)}{\lambda_{\mathrm{m}}\left(\boldsymbol{K}_{x x}\right) \lambda_{\mathrm{M}}\left(\boldsymbol{\Lambda}_{\dot{x} \dot{x}}\right)+\frac{1}{4}\left\|\boldsymbol{D}_{x \dot{x}}-\overline{\boldsymbol{\mu}}_{x \dot{x}}^{T}\right\|^{2}}:=\epsilon^{\mathrm{pow}},
$$

\footnotetext{
${ }^{7}$ Note that for all matrices $\boldsymbol{A}, \boldsymbol{E} \in \mathbb{R}^{n \times n},\|\boldsymbol{A} \boldsymbol{E}\| \leq\|\boldsymbol{A}\|\|\boldsymbol{E}\|$ and $\|\boldsymbol{A}+\boldsymbol{E}\| \leq\|\boldsymbol{A}\|+\|\boldsymbol{E}\|$ hold
} 
$\boldsymbol{S}_{\epsilon}^{\text {pow }}$ and $\boldsymbol{P}_{\epsilon}^{\text {pow }}$ are positive definite. The existence of $\epsilon^{\text {pow }}$ is ensured, since $\boldsymbol{\Lambda}_{\dot{x} \dot{x}}, \boldsymbol{K}_{x x}, \boldsymbol{D}_{x x} \succ 0$, and $\left\|\boldsymbol{D}_{\dot{x} \dot{x}}-\overline{\boldsymbol{\mu}}_{x \dot{x}}^{T}\right\|$ is upper bounded due to the established boundedness of $\overline{\boldsymbol{\mu}}(\boldsymbol{q}, \dot{\boldsymbol{q}})$.

In view of both (58) and (63), together with the existence of $\epsilon^{\text {ene }}$ and $\epsilon^{\text {pow }}$, one can conclude that there exists $\epsilon$ satisfying

$$
0<\epsilon<\min \left\{\epsilon^{\mathrm{ene}}, \epsilon^{\mathrm{pow}}\right\},
$$

such that $V_{\epsilon}(t, \boldsymbol{y})$ is positive definite and $\dot{V}_{\epsilon}(t, \boldsymbol{y})$ is negative definite in $\boldsymbol{y}$. Moreover, $\boldsymbol{P}_{\epsilon}^{\text {ene }}$ is bounded for bounded $\epsilon$, and $V_{\epsilon}(t, \boldsymbol{y})$ remains decrescent. Therefore, $V_{\epsilon}(t, \boldsymbol{y})$ is a valid strict Lyapunov function for the nonautonomous closed-loop system. Since both $V_{\epsilon}(t, \boldsymbol{y})$ and $\dot{V}_{\epsilon}(t, \boldsymbol{y})$ are also quadratic functions of $\boldsymbol{y}$, UES of $\boldsymbol{y}=\mathbf{0}$ can be straightforwardly established, e. g., through [40, Theorem 5.3.45] and [41, Theorem 4.10].

\section{Passivity Analysis for Physical Interaction}

One can employ $V(t, \boldsymbol{y})$ from (53) as a valid storage function for the analysis of passivity during physical interaction with the environment. During such an interaction, that is, $\boldsymbol{F}_{\dot{\boldsymbol{x}}}^{\text {ext }} \neq \mathbf{0}$, (54) can be written as

$$
\dot{V}=-\dot{\tilde{\boldsymbol{x}}}^{T} \boldsymbol{D} \dot{\tilde{\boldsymbol{x}}}+\dot{\tilde{\boldsymbol{x}}}^{T} \boldsymbol{F}_{\dot{\boldsymbol{x}}}^{\mathrm{ext}},
$$

and consequently,

$$
\dot{V}+\lambda_{\mathrm{m}}\left(\boldsymbol{D}_{\dot{x} \dot{x}}\right)\|\dot{\tilde{\boldsymbol{x}}}\|_{\boldsymbol{W}_{\dot{x}}}^{2} \leq \dot{\tilde{\boldsymbol{x}}}^{T} \boldsymbol{F}_{\dot{\boldsymbol{x}}}^{\mathrm{ext}}
$$

with $\lambda_{\mathrm{m}}\left(\boldsymbol{D}_{\dot{x} \dot{x}}\right)>0$. Therefore, the closed-loop system (39) is strictly output passive w.r.t. the input $\boldsymbol{F}_{\dot{x}}^{\text {ext }}$, the output $\dot{\tilde{\boldsymbol{x}}}$, and the storage function $V$ [43, Definition 2.2.8].

Remark 4: Note that for the regulation case $\left(\dot{\boldsymbol{x}}_{\mathrm{des}}=\mathbf{0}\right)$, the term $\dot{\tilde{\boldsymbol{x}}}^{T} \boldsymbol{F}_{\dot{\boldsymbol{x}}}^{\text {ext }}$ in (65) and (66) represents the physical power transmitted during interaction.

From (66) one can further conclude the boundedness of $\dot{\tilde{x}}$ in case of bounded inputs $\boldsymbol{F}_{\dot{x}}^{\text {ext }}$. Let $0<\theta<1$ be some positive

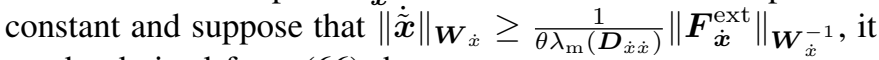
can be derived from (66) that

$$
\dot{V} \leq-(1-\theta) \lambda_{\mathrm{m}}\left(\boldsymbol{D}_{\dot{x} \dot{x}}\right)\|\dot{\tilde{\boldsymbol{x}}}\|_{\boldsymbol{W}_{\dot{x}}}^{2} .
$$

Therefore, the total energy $V$ will inevitably start to decrease when $\tilde{\tilde{x}}$ exceeds some limited bound defined with $\left\|\boldsymbol{F}_{\dot{x}}^{\text {ext }}\right\|_{\boldsymbol{W}_{\dot{x}}^{-1}}$. Since $\boldsymbol{\Lambda}(\boldsymbol{q})$ is uniformly bounded and the kinetic-energylike term $\frac{1}{2} \dot{\tilde{\boldsymbol{x}}}^{T} \boldsymbol{\Lambda} \dot{\tilde{\boldsymbol{x}}}$ can, at most, reach the value of $V$ (for $\tilde{\boldsymbol{x}}=\mathbf{0}$ ), one can conclude that $\dot{\tilde{\boldsymbol{x}}}$ is bounded under bounded $\boldsymbol{F}_{\dot{\boldsymbol{x}}}^{\text {ext }}$. Consequently, the boundedness of $\overline{\boldsymbol{\mu}}(\boldsymbol{q}, \dot{\boldsymbol{q}})$ can also be concluded for this case. The latter is a prerequisite for the following proof of input-to-state stability.

Remark 5: Note that the boundedness of $\overline{\boldsymbol{\mu}}(\boldsymbol{q}, \dot{\boldsymbol{q}})$ is restricted to both the case of bounded $\boldsymbol{F}_{\dot{x}}^{\text {ext }}$ and the case of free motion with $\boldsymbol{F}_{\dot{x}}^{\text {ext }}=\mathbf{0}$. The latter has already been shown in Section IV-C. For unbounded inputs, this conclusion cannot be made in general.

\section{E. Proof of Input-to-State Stability}

The following proposition related to ISS of the closed-loop system shows that $\boldsymbol{y}$ is bounded under bounded $\boldsymbol{F}_{\dot{\boldsymbol{x}}}^{\text {ext }}$, and it will approach the equilibrium as $\boldsymbol{F}_{\dot{x}}^{\text {ext }}$ tends to zero (see (68) below).
Proposition 2: Under the same conditions of Proposition 1, suppose that $\boldsymbol{F}_{\dot{\boldsymbol{x}}}^{\mathrm{ext}}$ is bounded during the entire execution of all tasks, that is, $\left\|\boldsymbol{F}_{\dot{\boldsymbol{x}}}^{\mathrm{ext}}\right\|_{\boldsymbol{W}^{-1}} \leq \rho \forall t \geq t_{0}$ with some positive constant $\rho$. Then, the closed-loop system (39) is (locally) inputto-state stable such that [44]

$$
\|\boldsymbol{y}\| \leq \beta\left(\left\|\boldsymbol{y}\left(t_{0}\right)\right\|, t-t_{0}\right)+\gamma\left(\sup _{t \geq t_{0}}\left\|\boldsymbol{F}_{\dot{x}}^{\mathrm{ext}}\right\|_{\boldsymbol{W}_{\dot{x}}^{-1}}\right),
$$

$\forall\left\|\boldsymbol{y}\left(t_{0}\right)\right\| \leq \rho$ and $\forall t \geq t_{0}$, where $\beta \in \mathcal{K} \mathcal{L}, \gamma \in \mathcal{K}$. In particular, a possible $\gamma(r)$ is given by

$$
\gamma(r):=\frac{\sqrt{\lambda_{\mathrm{M}}\left(\boldsymbol{P}_{\epsilon}^{\text {ene }}\right)}}{\sqrt{\lambda_{\mathrm{m}}\left(\boldsymbol{P}_{\epsilon}^{\text {ene }}\right)}} \frac{\sqrt{1+\eta \epsilon^{2}}}{\theta \lambda_{\mathrm{m}}\left(\boldsymbol{P}_{\epsilon}^{\text {pow }}\right)} r,
$$

where $\eta:=\max \left\{w_{\dot{x}, i} / w_{x, i}\right\}>0, \quad 0<\theta<1$ is a positive constant, and $\epsilon$ satisfies the condition (64) ensuring $\boldsymbol{P}_{\epsilon}^{\mathrm{ene}}, \boldsymbol{P}_{\epsilon}^{\text {pow }} \succ 0$.

Proof: The proof of Proposition 2 is based on the local version of [41, Theorem 4.19] and is conducted by showing that $V_{\epsilon}(t, \boldsymbol{y})$ is indeed a valid ISS-Lyapunov function [45]. As $\boldsymbol{P}_{\epsilon}^{\text {ene }}$ is positive definite and bounded, $V_{\epsilon}(t, \boldsymbol{y})$ satisfies

$$
\frac{1}{2} \lambda_{\mathrm{m}}\left(\boldsymbol{P}_{\epsilon}^{\mathrm{ene}}\right)\|\boldsymbol{y}\|^{2} \leq V_{\epsilon}(t, \boldsymbol{y}) \leq \frac{1}{2} \lambda_{\mathrm{M}}\left(\boldsymbol{P}_{\epsilon}^{\mathrm{ene}}\right)\|\boldsymbol{y}\|^{2} .
$$

For the case of physical interaction, i. e., $\boldsymbol{\tau}^{\mathrm{ext}} \neq \mathbf{0}$ (and $\left.\boldsymbol{F}_{\dot{\boldsymbol{x}}}^{\text {ext }} \neq \mathbf{0}\right)$, the time derivative of $V_{\epsilon}(t, \boldsymbol{y})$ can be written as

$$
\dot{V}_{\epsilon}\left(t, \boldsymbol{y}, \boldsymbol{F}_{\dot{\boldsymbol{x}}}^{\mathrm{ext}}\right)=-\boldsymbol{y}^{T} \boldsymbol{P}_{\epsilon}^{\mathrm{pow}} \boldsymbol{y}+\boldsymbol{y}^{T} \boldsymbol{F}_{\boldsymbol{y}}^{\mathrm{ext}}
$$

with $\boldsymbol{F}_{\boldsymbol{y}}^{\mathrm{ext}}:=\left[\begin{array}{ll}\epsilon \boldsymbol{F}_{\dot{\boldsymbol{x}}}^{\mathrm{ext}, T} \boldsymbol{W}_{x}^{-1 / 2} & \boldsymbol{F}_{\dot{\boldsymbol{x}}}^{\mathrm{ext}, T} \boldsymbol{W}_{\dot{x}}^{-1 / 2}\end{array}\right]^{T} \in \mathbb{R}^{2 n}$. Moreover, due to $\boldsymbol{P}_{\epsilon}^{\text {pow }} \succ 0, \dot{V}_{\epsilon}\left(t, \boldsymbol{y}, \boldsymbol{F}_{\dot{\boldsymbol{x}}}^{\text {ext }}\right)$ satisfies

$$
\dot{V}_{\epsilon}\left(t, \boldsymbol{y}, \boldsymbol{F}_{\dot{\boldsymbol{x}}}^{\mathrm{ext}}\right) \leq-\lambda_{\mathrm{m}}\left(\boldsymbol{P}_{\epsilon}^{\mathrm{pow}}\right)\|\boldsymbol{y}\|^{2}+\left|\boldsymbol{y}^{T} \boldsymbol{F}_{\boldsymbol{y}}^{\mathrm{ext}}\right| .
$$

Furthermore, it can be shown that

$$
\left|\boldsymbol{y}^{T} \boldsymbol{F}_{\boldsymbol{y}}^{\mathrm{ext}}\right| \leq\|\boldsymbol{y}\|\left\|\boldsymbol{F}_{\boldsymbol{y}}^{\mathrm{ext}}\right\| \leq \sqrt{1+\eta \epsilon^{2}}\|\boldsymbol{y}\|\left\|\boldsymbol{F}_{\dot{\boldsymbol{x}}}^{\mathrm{ext}}\right\|_{\boldsymbol{W}_{\dot{x}}^{-1}}
$$

where $\eta=\max \left\{w_{\dot{x}, i} / w_{x, i}\right\}$. It follows from (72)-(73) that $\forall\|\boldsymbol{y}\| \geq \frac{\sqrt{1+\eta \epsilon^{2}}}{\theta \lambda_{\mathrm{m}}\left(\boldsymbol{P}_{\epsilon}^{\text {oww }}\right)}\left\|\boldsymbol{F}_{\dot{\boldsymbol{x}}}^{\mathrm{ext}}\right\|_{\boldsymbol{W}_{\dot{\boldsymbol{x}}}^{-1}}$ with $0<\theta<1$,

$$
\dot{V}_{\epsilon}\left(t, \boldsymbol{y}, \boldsymbol{F}_{\dot{\boldsymbol{x}}}^{\mathrm{ext}}\right) \leq-(1-\theta) \lambda_{\mathrm{m}}\left(\boldsymbol{P}_{\epsilon}^{\text {pow }}\right)\|\boldsymbol{y}\|^{2} .
$$

Therefore, in view of (70) and (74), the closed-loop system is locally input-to-state stable w.r. t. the input $\boldsymbol{F}_{\dot{\boldsymbol{x}}}^{\mathrm{ext}}$ and the states $\boldsymbol{y}$, with a bounding class- $\mathcal{K}$ function $\gamma$ of $\sup _{t \geq t_{0}}\left\|\boldsymbol{F}_{\dot{\boldsymbol{x}}}^{\mathrm{ext}}\right\|_{\boldsymbol{W}_{\dot{x}}^{-1}}$ defined as (69).

\section{Simulations}

The theoretical properties of the proposed controller (38) with (33) are verified through simulations on a 6-DOF planar manipulator as illustrated in Fig. 2. The task hierarchy is designed with five priority levels:

- Level $1\left(m_{1}=2\right)$ : Translation of TCP in $x$ and $y$;

- Level $2\left(m_{2}=1\right)$ : Rotation of TCP about $z$;

- Level $3\left(m_{3}=1\right)$ : Rotation in joint 3 about $z$;

- Level $4\left(m_{4}=1\right)$ : Translation in joint 1 in $x$;

- Level $5\left(m_{5}=1\right)$ : Rotation in joint 5 about $z$.

The applied control gains are listed in Table I. As comparison 


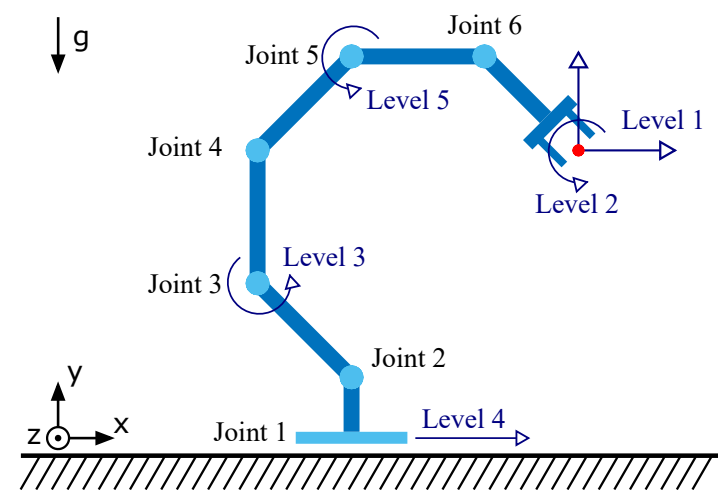

Fig. 2. Planar manipulator with 6 DOF used for numerical simulations. The model has one prismatic joint followed by five revolute joints, and it satisfies Assumptions 1 and 3. Gravity is simulated with $g=9.81 \mathrm{~m} / \mathrm{s}^{2}$. Each link is modeled as a massless bar with a centered point mass of $1 \mathrm{~kg}$. The base link has a length of $0.25 \mathrm{~m}$, all other links have a length of $0.5 \mathrm{~m}$. The Coriolis/centrifugal matrix is constructed by using the formulation presented in [28] which satisfies Assumption 2. The initial configuration is given by $\boldsymbol{q}_{0}=[0 \mathrm{~m}, 45 \mathrm{deg},-45 \mathrm{deg},-45 \mathrm{deg},-45 \mathrm{deg},-45 \mathrm{deg}]^{T}$.

TABLE I

CONTROL GAINS FOR THE SIMULATIONS

\begin{tabular}{l||c|c}
\hline \multicolumn{1}{c||}{ Lvl. } & $\begin{array}{c}\text { Sim. \#1 } \\
\text { stiffness/damping }\end{array}$ & $\begin{array}{c}\text { Sim.\#2 and \#3 } \\
\text { stiffness/damping }\end{array}$ \\
\hline 1 & $(200,200) \frac{\mathrm{N}}{\mathrm{m}} /(10,10) \frac{\mathrm{Ns}}{\mathrm{m}}$ & $(400,400) \frac{\mathrm{N}}{\mathrm{m}} /(50,50) \frac{\mathrm{Ns}}{\mathrm{m}}$ \\
2 & $50 \frac{\mathrm{Nm}}{\mathrm{rad}} / 5 \frac{\mathrm{Nms}}{\mathrm{rad}}$ & $300 \frac{\mathrm{Nm}}{\mathrm{rad}} / 30 \frac{\mathrm{Nms}}{\mathrm{rad}}$ \\
3 & $50 \frac{\mathrm{Nm}}{\mathrm{rad}} / 5 \frac{\mathrm{Nms}}{\mathrm{rad}}$ & $100 \frac{\mathrm{Nm}}{\mathrm{rad}} / 10 \frac{\mathrm{Nms}}{\mathrm{rad}}$ \\
4 & $100 \frac{\mathrm{N}}{\mathrm{m}} / 10 \frac{\mathrm{Ns}}{\mathrm{m}}$ & $250 \frac{\mathrm{N}}{\mathrm{m}} / 30 \frac{\mathrm{Ns}}{\mathrm{m}}$ \\
5 & $50 \frac{\mathrm{Nm}}{\mathrm{rad}} / 5 \frac{\mathrm{Nms}}{\mathrm{rad}}$ & $100 \frac{\mathrm{Nm}}{\mathrm{rad}} / 10 \frac{\mathrm{Nms}}{\mathrm{rad}}$ \\
\hline
\end{tabular}

to the proposed controller, the classical feedback-linearizationbased controller with (40) is evaluated using the same set of control gains from Table I. Furthermore, two types of desired task-space inertias are implemented and analyzed for the feedback linearization

$$
\text { I }: \boldsymbol{\Lambda}_{i, \mathrm{des}}=\boldsymbol{\Lambda}_{i, \mathrm{kin}}\left(\boldsymbol{q}_{0}\right) ; \quad \text { II }: \boldsymbol{\Lambda}_{i, \mathrm{des}}=\boldsymbol{I} .
$$

Herein, $\boldsymbol{q}_{0}$ denotes the initial joint positions, and $\boldsymbol{\Lambda}_{i, \mathrm{kin}}\left(\boldsymbol{q}_{0}\right)$ is computed through (47). The term $\boldsymbol{\Lambda}_{i, \mathrm{kin}}\left(\boldsymbol{q}_{0}\right)$ can be interpreted as the natural task-space inertia in the initial configuration when a single task is performed.

Simulation \#1 (convergence): The first simulations aim at verifying the convergence properties of the proposed controller. All five levels are assigned with smooth position trajectories as shown in Fig. 3, which can cause the system to undergo large configuration changes. At $t=0 \mathrm{~s}$, the trajectories are deviated from the zero points in order to induce initial task-space position errors. Fig. 4 depicts the Euclidean norm of the tracking errors on each level. Note that the norm values are physically consistent as task coordinates on each level have the same unit. One can observe that the proposed controller provides the desired exponential stability and features a similar convergence rate as the feedback linearization of Type I. Moreover, because of the full compensation of top-down disturbances, the transient behavior of lower-level tasks is not
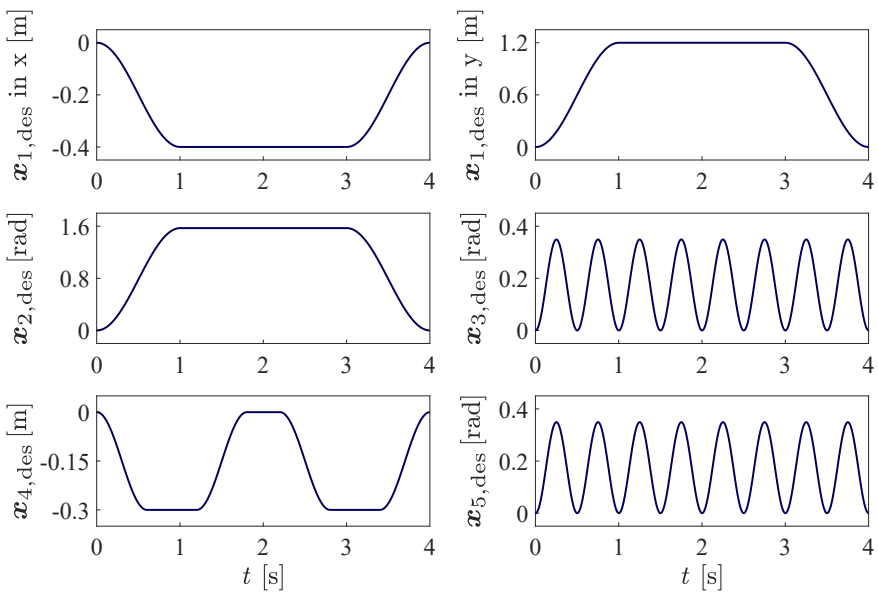

Fig. 3. Simulation \#1: Desired position trajectories on all five levels. The zero points of the level-specific trajectories correspond to the respective initial configuration as depicted in Fig. 2.
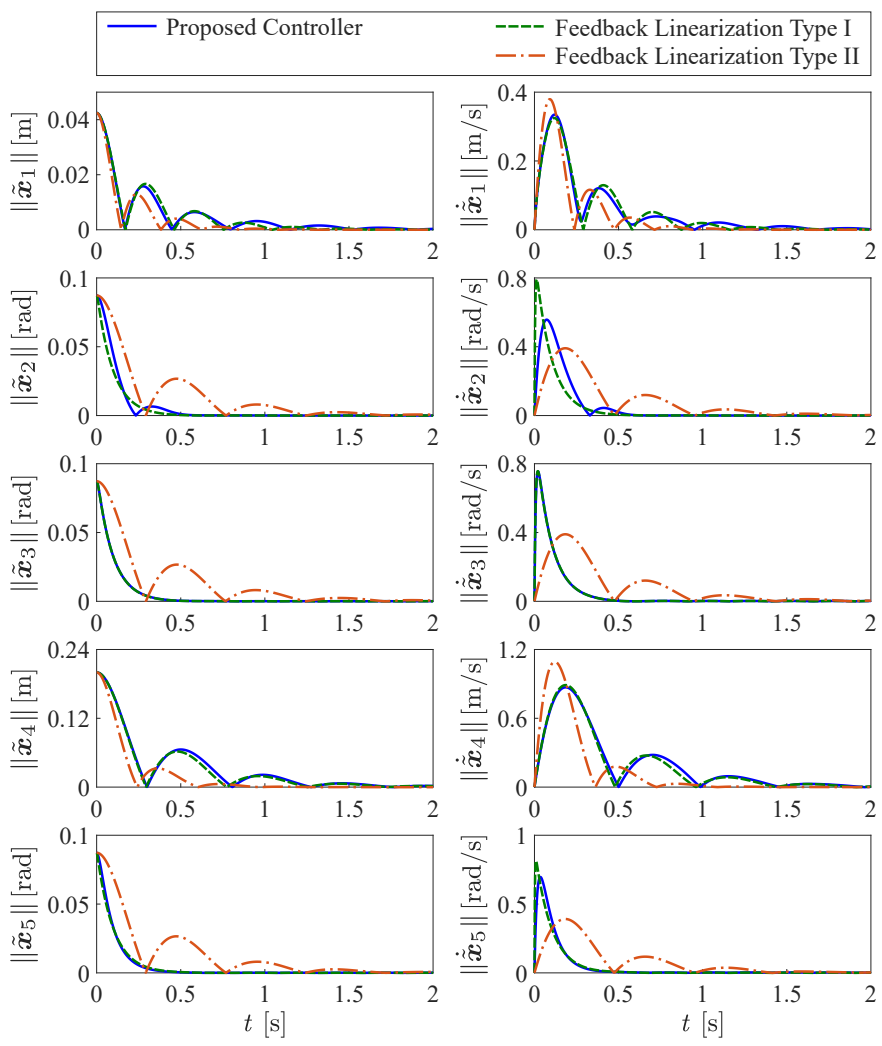

Fig. 4. Simulation \#1: Comparison of the convergence behavior in free motion between the proposed controller and the feedback linearization of Type I and II. Because all tracking errors can converge within $2 \mathrm{~s}$, the results after $t=2 \mathrm{~s}$ are omitted for clarity.

affected (or only marginally affected due to dependencies on joint states) by higher-level tasks. For example, the tracking errors on levels 2,3 , and 5 can decay even faster to zero than the ones on level 1.

Simulation \#2 (performance robustness): In order to compare their robustness properties, the closed-loop time response for each controller is evaluated 1000 times in the presence of parameter variations and unmodeled friction effects. Specifically, the link mass used in the simulated dynamics is varied 


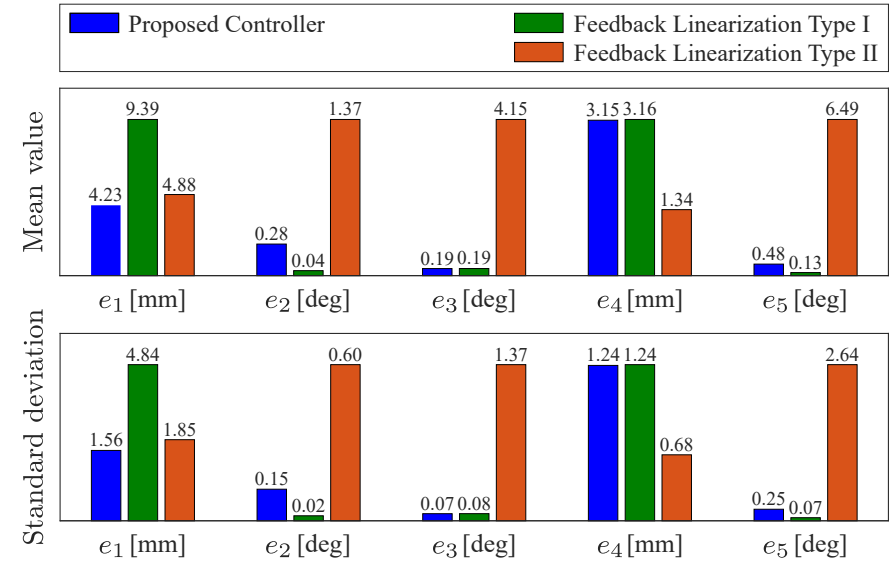

Fig. 5. Simulation \#2: Mean values and standard deviations of the RMS errors computed from 1000 evaluations for each controller. Link mass and joint friction coefficients are varied by the respective uniform distributions.

for all links together by means of a uniform distribution over $\pm 15 \%$ of the nominal value. Moreover, all joints are affected by unmodeled viscous friction with uniformly distributed coefficients between 0 and $0.1 \mathrm{Nms} / \mathrm{rad}$ for the revolute joints or Ns/m for the base joint, respectively. As justified in [46], the uniform distribution can be seen as the worst-case distribution for the robustness analysis when statistical descriptions of the uncertainty are unavailable. The desired trajectories depicted in Fig. 3 are reused but without including any initial task-space errors. The tracking performance of one single evaluation is quantified by the root mean square (RMS) values of $\tilde{\boldsymbol{x}}_{i}$, that is, $e_{i}=\sqrt{\frac{1}{T_{s}} \int_{0}^{T_{s}} \tilde{\boldsymbol{x}}_{i}^{T} \tilde{\boldsymbol{x}}_{i} \mathrm{~d} t}$ with $T_{s}=4 \mathrm{~s}$ being the total simulation time. The statistical results of all evaluations are summarized in Fig. 5 which provides a comparison of performance robustness of the controllers. It can be observed that the proposed controller achieves the best tracking performance on level 1 and similar performance on levels 3 and 4 as the feedback linearization of Type I.

On the other hand, the proposed controller can result in considerably larger position errors on levels 2 and 5 than the feedback linearization of Type I. The reasons are two-fold: a) the impact of $\boldsymbol{\Lambda}_{i} \boldsymbol{\Lambda}_{i \text {,des }}^{-1}$ in the feedback linearization is reduced to a scalar factor when the subtask is only one-dimensional, as mentioned in Section III-B1; b) in order to comply with the strict task hierarchy, the controllability of these two subtasks is largely limited. To elaborate on the latter, the eigenvalues of $\boldsymbol{\Lambda}_{i}$ and $\boldsymbol{\Lambda}_{i, \text { kin }}\left(\boldsymbol{q}_{0}\right)$ on all subordinated levels $i=2 \ldots 5$ during the last evaluation are plotted in Fig. 6. Based on the implemented task hierarchy, one would expect that the subtasks on levels 2 and 5 are more likely to compete with the main task. This is confirmed by the observation that $\lambda_{i}$ is much larger than $\lambda_{i, \text { kin }}$ on levels 2 and 5, also in the initial configuration, whereas they have comparable magnitudes on levels 3 and 4 . Therefore, on levels 2 and 5 , the factor $\boldsymbol{\Lambda}_{i} \boldsymbol{\Lambda}_{i \text {, kin }}^{-1}\left(\boldsymbol{q}_{0}\right)=\lambda_{i} / \lambda_{i, \text { kin }}>1$ aggressively increases the PD control gains (i. e., both stiffness and damping) and can, in turn, result in better tracking performance. However, this enhancement of performance with the feedback linearization has to be paid by increased risk of exceeding the feasible force/torque limits of actuators.
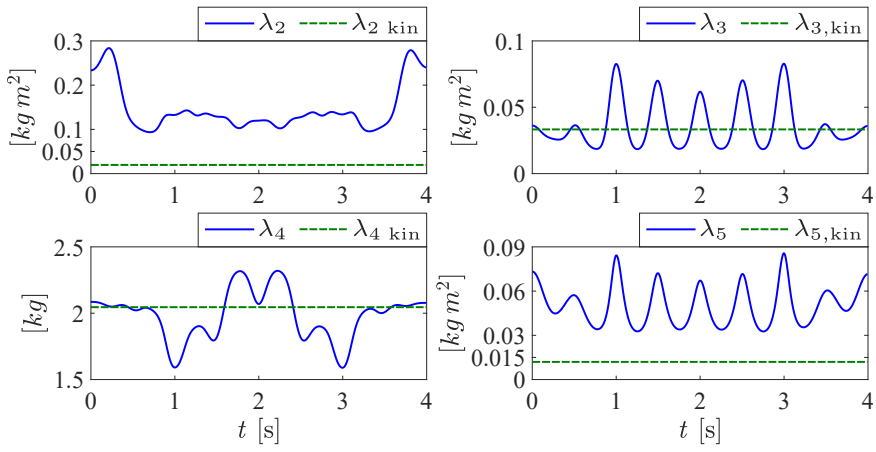

Fig. 6. Simulation \#2: Eigenvalues of hierarchy-consistent and non-consistent task-space inertias $\left(\boldsymbol{\Lambda}_{i}\right.$ and $\left.\boldsymbol{\Lambda}_{i, \mathrm{kin}}\left(\boldsymbol{q}_{0}\right)\right)$ on levels $i=2 \ldots 5$. Since all subtasks are one-dimensional, $\lambda_{i}$ and $\lambda_{i, \text { kin }}$ are the respective task-space inertias themselves.

Simulation \#3 (physical interaction): To examine the physical interaction behavior of the proposed controller, external task-space forces/torques are applied separately on all levels of the task hierarchy. Fig. 7 shows the applied external forces/torques together with the reference trajectories. To analyze the robustness issues of external force feedback, the classical momentum-based observer of $\tau^{\text {ext }}$ [47], [48] is implemented for the simulations. Roughly speaking, the observer can provide a first-order filtered estimate of $\tau^{\text {ext }}$, that is, $\dot{\boldsymbol{r}}=-\boldsymbol{K}_{O}\left(\boldsymbol{r}-\boldsymbol{\tau}^{\text {ext }}\right)$ with $\boldsymbol{r}$ being the observer output, the socalled residual vector, and $\boldsymbol{K}_{O} \succ 0$ a diagonal gain matrix of the observer. The task-space position errors with the proposed controller and the feedback linearization of Type I during physical interaction are plotted in Fig. 8. It can be observed that when using the (unrealistic) perfect knowledge of $\tau^{\text {ext }}$, both controllers can feature the desired decoupled impedance behavior on the individual levels. Under cross-couplings in $\boldsymbol{F}_{\dot{\boldsymbol{x}}}^{\text {ext }}$ due to estimation errors, the proposed controller shows better performance on level 1 and comparable performance as the feedback linearization on all other levels. It is noticeable that the performance of the feedback linearization deteriorates in inactive phases of interactions on level 1, especially during fast changes of configuration, e.g., for $3 \mathrm{~s}<t<4 \mathrm{~s}$.

\section{EXPERIMENTS}

The proposed approach is validated and compared with the HPD+ and the feedback linearization in two sets of experiments: trajectory tracking in free motion (Exp.\#1) and under physical interaction (Exp.\#2). All experiments are conducted on a 7-DOF torque-controlled KUKA LWR IV+. The task hierarchy comprises three priority levels. The main task is defined by a translational Cartesian impedance at the TCP with $m_{1}=3$. On the second level, an impedance of the TCP orientation with $m_{2}=3$ is considered, where the PD control term is implemented via a quaternion-based potential function to avoid representation singularities [49]. Joint impedance of the first joint with $m_{3}=1$ is assigned to the lowest level. The applied control gains are given in Table II.

To provide a direct comparison between the proposed approach and the state of the art, the feedback linearization of type I (i.e., $\boldsymbol{\Lambda}_{i, \mathrm{des}}=\boldsymbol{\Lambda}_{i \text {,kin }}\left(\boldsymbol{q}_{0}\right)$ ), and the HPD+ approach 

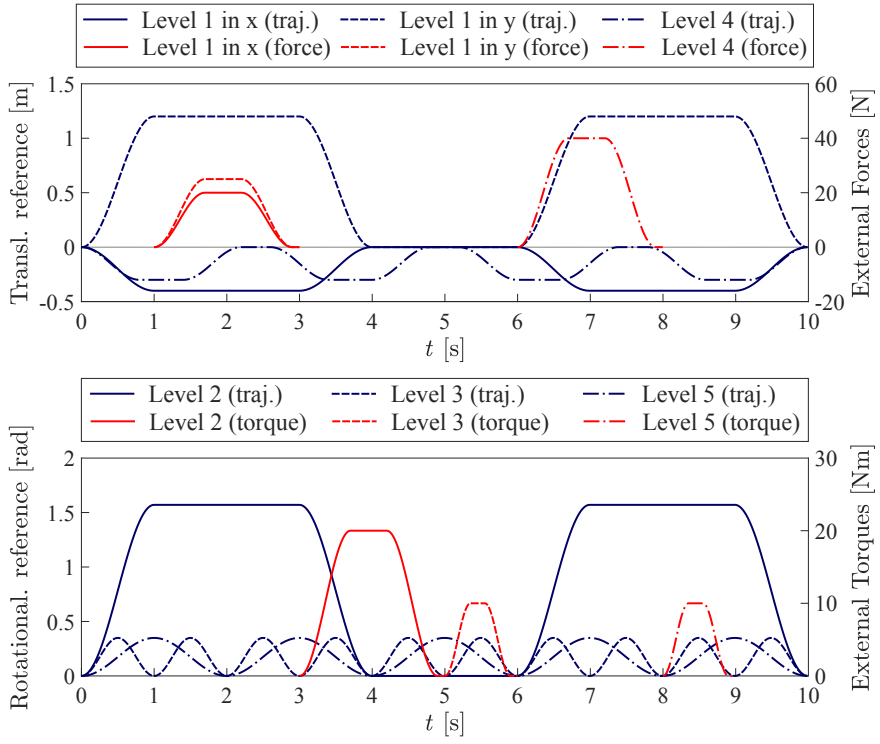

Fig. 7. Simulation \#3: Reference trajectories (blue) and applied external taskspace forces/torques (red) on all levels of the task hierarchy.

TABLE II

CONTROL GAINS FOR THE EXPERIMENTS

\begin{tabular}{l||c|c}
\hline Lvl. & $\begin{array}{c}\text { Exp.\#1 } \\
\text { stiffness/damping ratio }\end{array}$ & $\begin{array}{c}\text { Exp.\#2 } \\
\text { stiffness/damping ratio }^{\star}\end{array}$ \\
\hline 1 & $1500 \frac{\mathrm{N}}{\mathrm{m}} / 0.7$ & $500 \frac{\mathrm{N}}{\mathrm{m}} / 0.7$ \\
2 & $200 \frac{\mathrm{Nm}}{\mathrm{rad}} / 0.7$ & $100 \frac{\mathrm{Nm}}{\mathrm{rad}} / 0.7$ \\
3 & $300 \frac{\mathrm{Nm}}{\mathrm{rad}} / 0.7$ & $200 \frac{\mathrm{Nm}}{\mathrm{rad}} / 0.7$ \\
\hline
\end{tabular}

${ }^{\star}$ Realized through the double diagonalization approach [50].

without the optional compensation (26) are considered and tested using the same control gains from Table II. The stiffness values are designed according to the main goal of the tasks, i. e., a moderate stiffness for dynamic trajectory tracking with limited contact forces, and a low stiffness for compliant physical interaction with the environment. Moreover, in order to feature a comparable amount of damping effect in all approaches, damping ratios instead of constant damping values are intentionally applied by using the double diagonalization method [50]. The generalized external force $\tau^{\text {ext }}$ is estimated by means of the widely used momentum-based observer [47], [48] as mentioned in the simulation section, and then mapped to the task space to acquire $\boldsymbol{F}_{\dot{x}}^{\text {ext }}$, which is needed by the controller of the proposed approach and the feedback linearization.The diagonal entries of the observer gain matrix $\boldsymbol{K}_{O}$ are selected as $30 \mathrm{~s}^{-1}$ throughout the experiments. That parameterization corresponds to the practical range of $25 \mathrm{~s}^{-1}$ to $75 \mathrm{~s}^{-1}$ as analyzed in [51].

Experiment \#1 (free motion): In this experiment a translational figure-eight trajectory with high speed profile is applied to the TCP in $y$ - and $z$-directions, while a synchronized sinus trajectory is assigned to the TCP in $x$-direction. Each cycle of the figure-eight trajectory is completed within $2.5 \mathrm{~s}$ (in a $0.75 \mathrm{~m} \times 0.22 \mathrm{~m}$ region in the $y z$-plane), and the maximum desired linear velocity of the TCP is about $0.86 \mathrm{~m} / \mathrm{s}$. Moreover, compatible rotational trajectories are designed for the
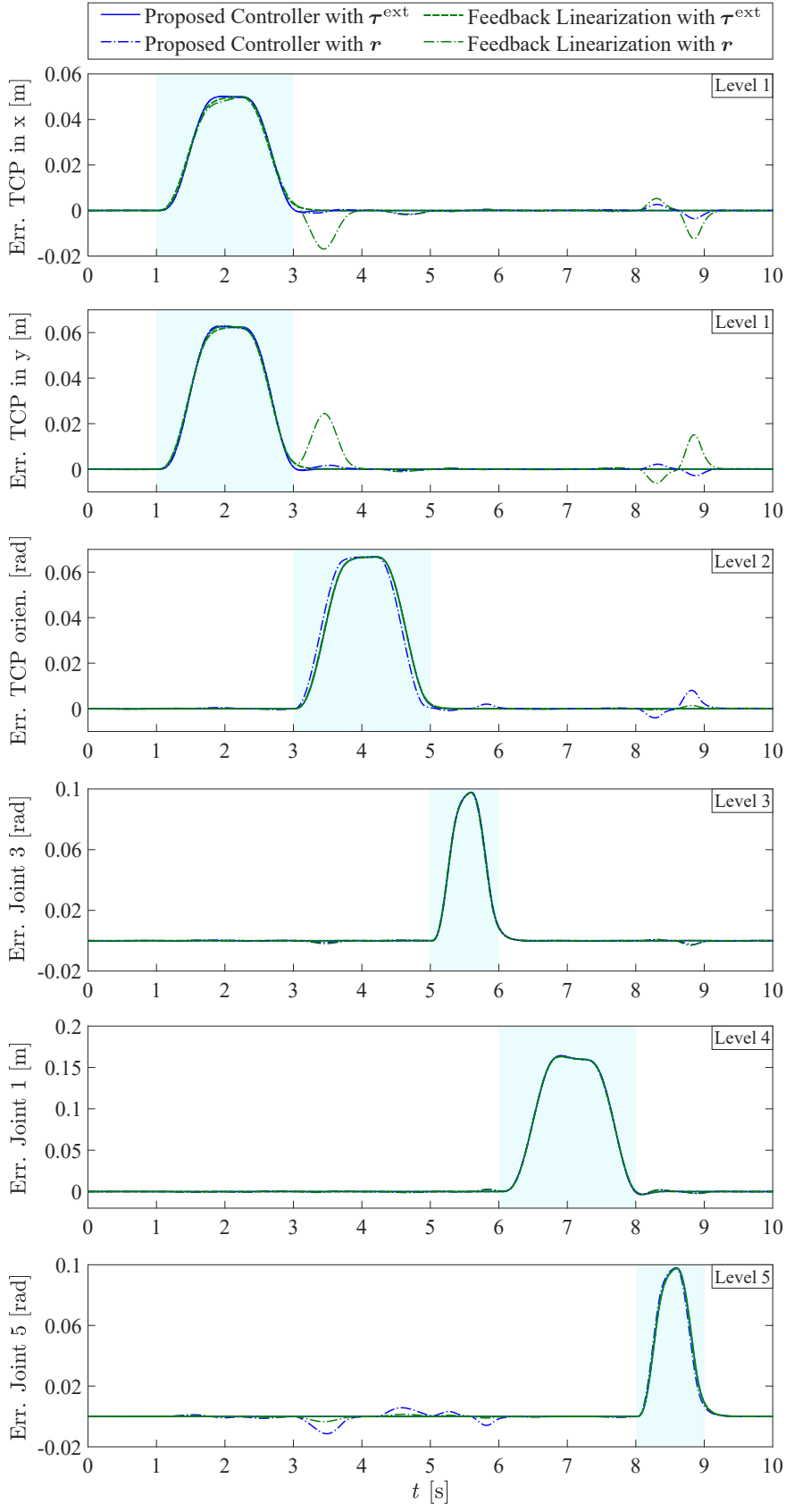

Fig. 8. Simulation \#3: Task-space position errors induced by external interactions during the trajectory-tracking. The shaded areas mark the time intervals of active interactions on the respective hierarchy levels.

secondary tasks on levels 2 and 3. The initial configuration and the desired trajectories of all tasks are presented in Fig. 9. The absolute task-space tracking errors using different controllers are depicted in Fig. 10. One can observe that the best performance is achieved with the HPD+, whereas the performances of the proposed approach and the feedback linearization deteriorate mainly due to the estimation errors of the momentum-based observer. As this observer is modelbased, it will mistakenly treat the unmatched dynamics caused by modeling errors as external forces/torques exerted by the environment, especially when the system undergoes highly dynamic motions. In fact, the estimated total external force on 


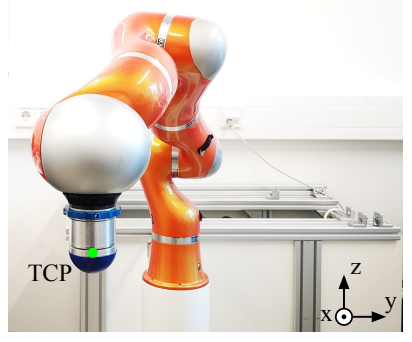

(a)

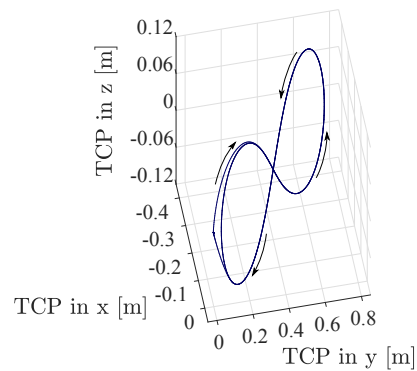

(c)
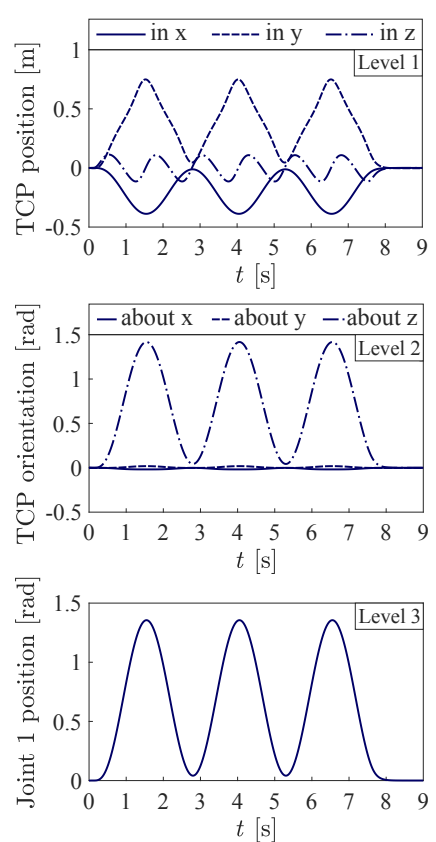

(b)
Fig. 9. Experiment \#1: a) initial joint configuration described by $\left.\boldsymbol{q}_{0}=[-0.2,0.9,-0.21,-1.1,0.20,1.14,0]^{T} \mathrm{rad} ; \mathrm{b}\right)$ desired trajectories of all hierarchy levels; c) figure-eight trajectory of TCP position (level 1).
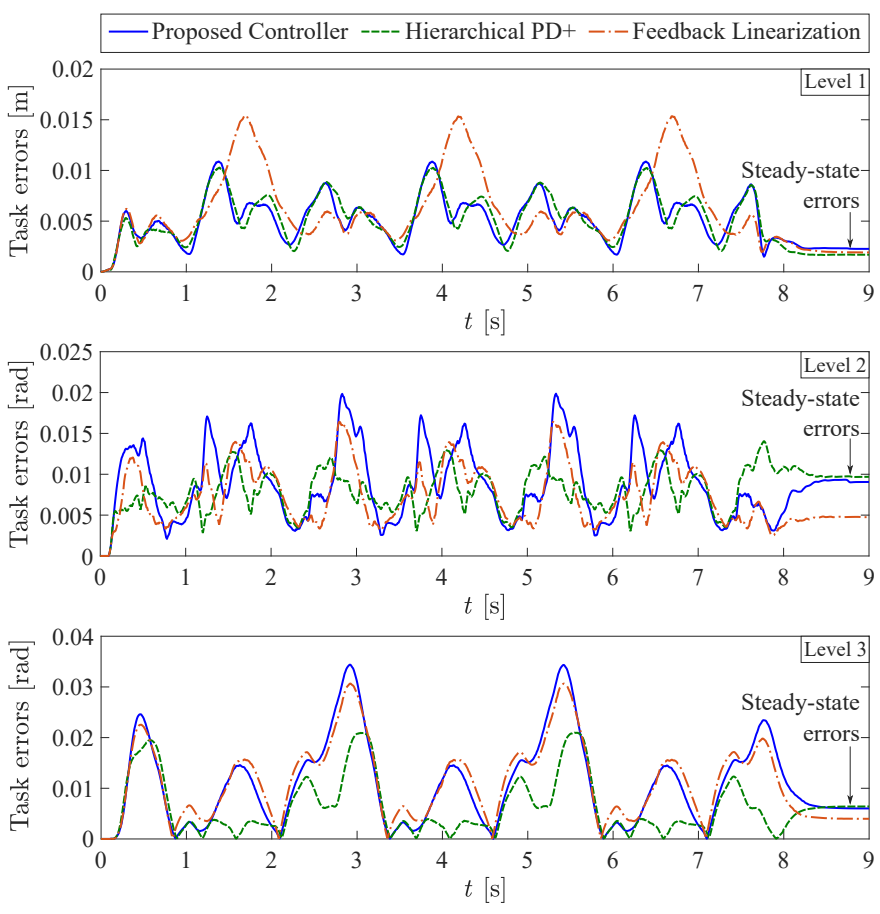

Fig. 10. Experiment \#1: Absolute task-space tracking errors of different controllers. The orientation error of the TCP (level 2) is represented by the absolute angle between the current and the desired TCP frames.

level 1, which is the Euclidean norm of the estimated external forces at the TCP in all three directions, has a maximum value of about $25 \mathrm{~N}$, while the Euclidean norm of the estimated external torques on levels 2 and 3 is at most $2.5 \mathrm{Nm}$ and $4.5 \mathrm{Nm}$, respectively. However, it can be seen that the proposed approach can still achieve similar performance on level 1 as the
TABLE III

EXPERIMENT \# 1: ABSOLUTE MAXIMUM (AM) AND ROOT MEAN SQUARED (RMS) TASK ERRORS USING DIFFERENT APPROACHES.

\begin{tabular}{l||cc|cc|cc}
\hline \multirow{2}{*}{ Approach } & \multicolumn{2}{|c|}{ Err. on L1 [m] } & \multicolumn{2}{c|}{ Err. on L2 $[\mathrm{rad}]$} & \multicolumn{2}{c}{ Err. on L3 [rad] } \\
\cline { 2 - 7 } & AM & RMS & AM & RMS & AM & RMS \\
\hline Proposed & 0.0109 & 0.0056 & 0.0199 & 0.0098 & 0.0344 & 0.0137 \\
\hline HPD+ & 0.0103 & 0.0057 & 0.0141 & 0.0085 & 0.0210 & 0.0084 \\
\hline FL $^{\star}$ & 0.0154 & 0.0071 & 0.0164 & 0.0080 & 0.0307 & 0.0134 \\
\hline
\end{tabular}

* Feedback Linearization.

HPD+. By contrast, the maximum task error on level 1 with the feedback linearization is about $40-50 \%$ larger than the ones with the other two approaches. This comparison confirms the theoretical result from Section III-B2 that the proposed approach is less sensitive against the estimation/measurement errors of $\boldsymbol{F}_{\dot{\boldsymbol{x}}}^{\text {ext }}$ on the first priority level. It is noticeable that the proposed approach has the largest task errors on levels 2 and 3. Nevertheless, the maximum overshoots with the proposed controller and the feedback linearization, relative to the respective steady-state errors, have similar magnitudes on these two levels. The absolute maximum and root mean squared task errors of different approaches are provided in Table III.

Experiment \#2 (physical interaction): Trajectory tracking tasks are performed under unknown physical interactions on the individual hierarchy levels. The experimental setup and the desired trajectories are depicted in Fig. 11. A line trajectory is given on level 1 to move the TCP towards a wooden plate which is fixed through aluminum profiles in the $x z$ plane. After colliding with the plate, the end-effector is held at the contact point for about $1.5 \mathrm{~s}$ (holding phase), and then it is commanded to move along the plate in $x$-direction for about $1.4 \mathrm{~s}$ (sliding phase) before moving back to the initial pose. Additionally, virtual external torques are applied on levels 2 and 3 through motor commands in order to provide reproducible interaction conditions for different experimental trials.

Fig. 12 shows the absolute task errors and the Euclidean norm of estimated external task-space forces/torques. The shaded areas represent the time intervals when the levelspecific interaction occurs, i. e. when $\boldsymbol{F}_{\dot{\boldsymbol{x}}_{i}}^{\text {ext }}$ is active on level $i$. Thus, the task deviations within such areas are expected as a result of the desired impedance behavior. It can be identified that the proposed approach essentially shows superiority in terms of compensating for couplings across the hierarchy levels. In the case of feedback linearization, large errors of the main task can be observed during free motion phases on level 1, as highlighted by the peak values in diagram 1 of Fig. 12. These main task errors also give rise to substantial deviations on level 2 as seen in diagram 3, which indicates the insufficient compensation of the top-down couplings with the feedback linearization in practice. As aforementioned, a virtual torque of $-15 \mathrm{Nm}$ is applied to the first joint from $5.5 \mathrm{~s}$ to $8 \mathrm{~s}$ (level 3), and another one of $10 \mathrm{Nm}$ is exerted at the TCP about the $x$-axis from $12.5 \mathrm{~s}$ to $15 \mathrm{~s}$ (level 2), see diagrams 4 and 6 , respectively. When the HPD+ is chosen, 


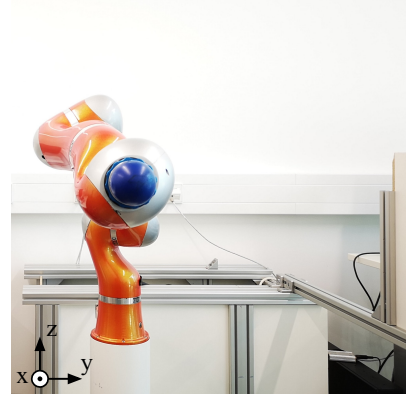

(a)

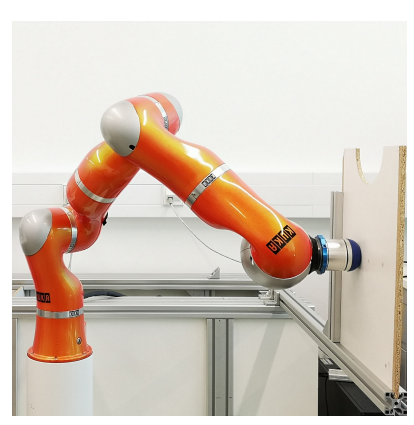

(b)
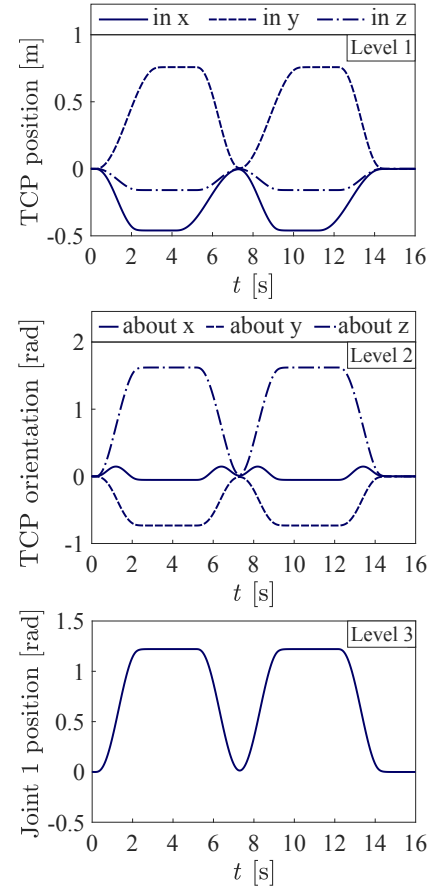

(c)

Fig. 11. Experiment \#2: a) initial joint configuration described by $\boldsymbol{q}_{0}=[-0.44,0.9,0.68,-1.1,0.2,-0.4,1.6]^{T} \mathrm{rad}$; b) configuration during the holding phase of the physical contact; c) desired trajectories of all hierarchy levels.

the virtual interaction on level 3 induces considerable task errors on both higher levels, and the one on level 2 causes the highest peak on level 1 during free motion. This is due to the fact that the considered HPD+ controller does not incorporate compensation of the bottom-up couplings in $\boldsymbol{F}_{\dot{\boldsymbol{x}}}^{\text {ext }}$.

The last issue of Exp. \#2 to be reported is the instability with the feedback linearization, when a higher gain of $\boldsymbol{K}_{O}$ is chosen to achieve faster convergence of the observer. Fig. 13 shows the results for $\boldsymbol{K}_{O}=70 \mathrm{~s}^{-1}$, which is still within the common and practical range for such lightweight robots [51]. At $t \approx 2 \mathrm{~s}$, the feedback linearization destabilizes the system before any physical interaction occurs. Note that oscillations arise on all hierarchy levels with the feedback linearization and the redundant results on levels 2, 3 are omitted here. During contact with the plate, the oscillations continue to exist at a frequency of about $20 \mathrm{~Hz}$. As with the proposed approach, no appreciable deterioration can be observed under such a high gain of the observer. Note that before $t=5.5 \mathrm{~s}$ no physical interactions are applied on the lower levels. Therefore, the performance of the HPD+ on level 1 during this period can be viewed as the nominal one for the proposed approach, since the HPD+ does not use any external force/torque feedback.

\section{CONCLUSION AND Future Works}

Multi-task control on kinematically redundant robots is often implemented through the application of dynamically consistent task hierarchies. One of the most commonly applied approaches is the operational space formulation, which feedback-linearizes the system, but it requires the control
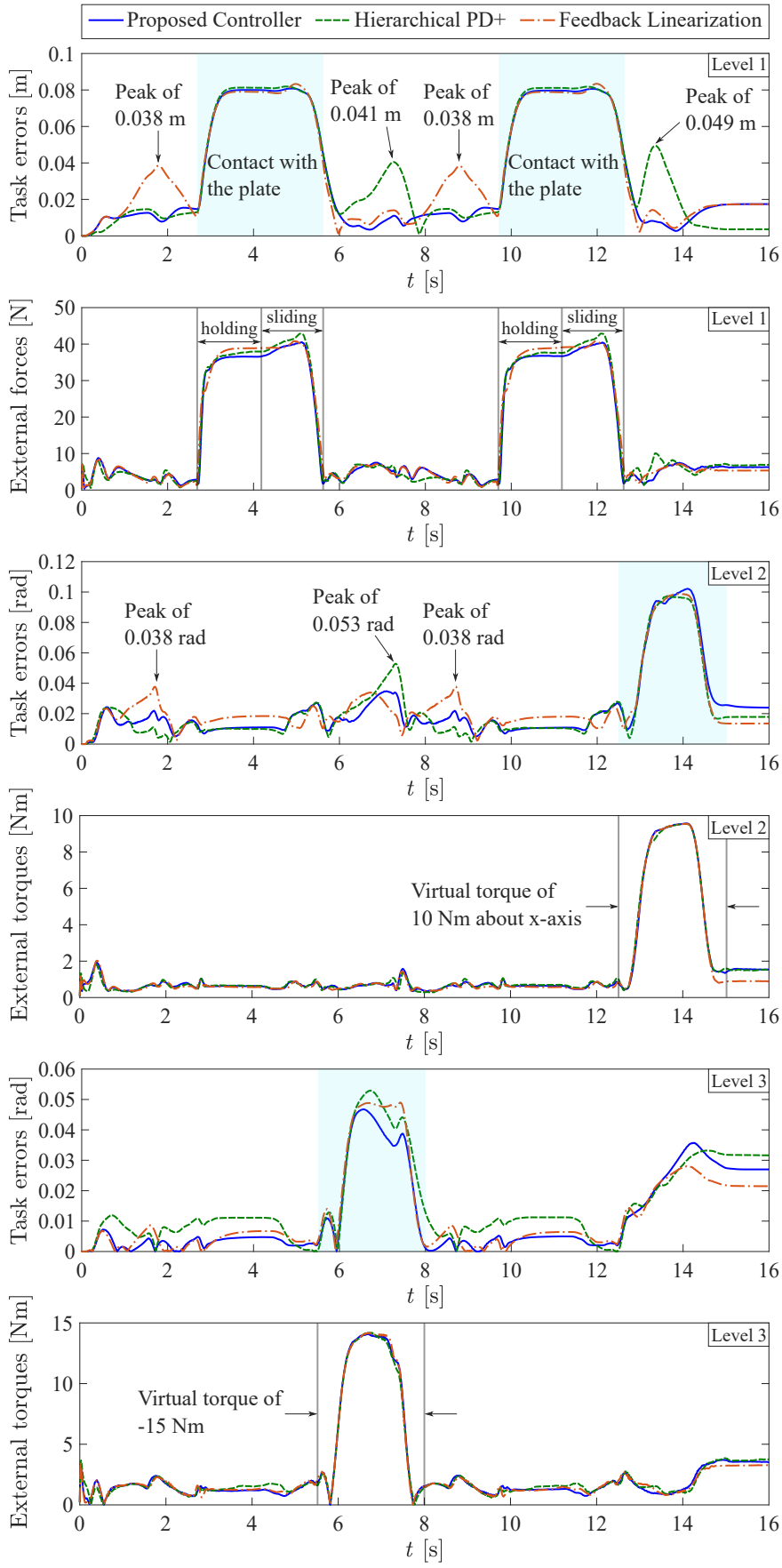

Fig. 12. Experiment \#2: Absolute task-space tracking errors (diagrams 1, 3, 5) and Euclidean norm of estimated external task-space forces/torques (diagrams $2,4,6)$ on all hierarchy levels. The time intervals of occurrences of levelspecific interactions are marked by the blue shaded rectangles.

feedback of external forces and the active modification of inertial properties. While being theoretically superior, robustness problems often arise in practice. An alternative approach is the hierarchical PD+ controller which conserves the natural inertia and avoids the feedback of external forces, and it turns out to be more robust in practice. However, it features nonlinear closed-loop dynamics and leads to external-force couplings across the hierarchies. In this work, we have proposed a passivity-based approach that combines the advantages of both worlds: feedback of external forces to improve the physical 



Fig. 13. Experiment \#2: Instability of the feedback linearization with $\boldsymbol{K}_{O}=70 \mathrm{~s}^{-1}$

interaction behavior, and avoidance of inertia shaping to preserve the natural dynamics of the system. The approach was theoretically analyzed in terms of stability and passivity properties. Simulations on a planar manipulator and experiments on a standard torque-controlled robot have been performed and complemented by the direct comparisons with the hierarchical $\mathrm{PD}+$ and the OSF.

Suitable measures to cope with kinematic and algorithmic singularities are often needed when applying task-space controllers. Since the controller can only react locally to any encountered singularities based on the current state of the system, it is commonly required that the task and motion planners provide fully compatible task objectives and kinematically feasible reference trajectories [7], [17]. On the other hand, discontinuous rank-changes of the Jacobian matrices can arise near to singular configurations. To deal with this and enhance its singularity robustness, our approach can be straightforwardly extended with well-known techniques, such as damped least squares methods [52] and projection shaping [53], which will be considered in future works.

The proposed approach cannot be directly applied for whole-body control of humanoids, which are usually modeled as an under-actuated, floating-base system. In future works, we will account for contact constraints by enforcing the execution of each task to comply with both strict hierarchies and constraints, as achieved in [10], [12]. By this means, the proposed control framework can be made applicable to floating-base robots as well.

\section{APPENDIX A}

The following propositions show that the invertibility of $\boldsymbol{J}$ implies the invertibility of $\overline{\boldsymbol{J}}$.

1) Proposition 3: If $\boldsymbol{J}$ is nonsingular, then $\overline{\boldsymbol{J}}_{i}$ for $i=1 \ldots r$ have full row rank.

Proof: Let $\boldsymbol{J}_{i}^{\text {aug }}$ denote the augmented Jacobian matrix down to the $i$-th hierarchy level, i. e., $\boldsymbol{J}_{i}^{\text {aug }}:=\left[\begin{array}{lll}\boldsymbol{J}_{1}^{T} & \ldots & \boldsymbol{J}_{i}^{T}\end{array}\right]^{T}$ and consequently $\boldsymbol{J}_{r}^{\text {aug }}=\boldsymbol{J}$. It follows from

$$
\boldsymbol{J}_{i}^{\text {aug }} \boldsymbol{N}_{i}^{T}=\left[\begin{array}{c}
\boldsymbol{J}_{i-1}^{\text {aug }} \boldsymbol{N}_{i}^{T} \\
\boldsymbol{J}_{i} \boldsymbol{N}_{i}^{T}
\end{array}\right]=\left[\begin{array}{c}
\mathbf{0} \\
\overline{\boldsymbol{J}}_{i}
\end{array}\right] \quad \text { for } i>1
$$

and $\boldsymbol{J}_{1}^{\text {aug }} \boldsymbol{N}_{1}^{T}=\overline{\boldsymbol{J}}_{1}$ that $\operatorname{rank}\left(\boldsymbol{J}_{i}^{\text {aug }} \boldsymbol{N}_{i}^{T}\right)=\operatorname{rank}\left(\overline{\boldsymbol{J}}_{i}\right) \leq m_{i}$. On the other hand, Sylvester's inequality states

$$
\operatorname{rank}\left(\boldsymbol{J}_{i}^{\text {aug }} \boldsymbol{N}_{i}^{T}\right) \geq \operatorname{rank}\left(\boldsymbol{J}_{i}^{\text {aug }}\right)+\operatorname{rank}\left(\boldsymbol{N}_{i}^{T}\right)-n .
$$

If $\boldsymbol{J}$ is nonsingular, then $\operatorname{rank}\left(\boldsymbol{J}_{i}^{\text {aug }}\right)=\sum_{j=1}^{i} m_{j}$ holds obviously. Moreover, the rank of any null-space projector is $\operatorname{rank}\left(\boldsymbol{N}_{i}^{T}\right)=n-\sum_{j=1}^{i-1} m_{j}$. Consequently (76) can be simplified to $\operatorname{rank}\left(\boldsymbol{J}_{i}^{\text {aug }} \boldsymbol{N}_{i}^{T}\right) \geq m_{i}$. Together with the inequality inferred from (75), one obtains $\operatorname{rank}\left(\boldsymbol{J}_{i}^{\text {aug }} \boldsymbol{N}_{i}^{T}\right)=m_{i}$. That, in turn, leads to $\operatorname{rank}\left(\overline{\boldsymbol{J}}_{i}\right)=m_{i}$, as shown in (75).

2) Lemma 1: [33] $\boldsymbol{N}_{i}^{T}=\boldsymbol{I}-\boldsymbol{J}_{i-1}^{\text {aug, } \boldsymbol{M}+} \boldsymbol{J}_{i-1}^{\text {aug }}$ for $i=2 \ldots r$.

3) Proposition 4: If $\overline{\boldsymbol{J}}_{i}$ for $i=1 \ldots$ have full row rank, then $\overline{\boldsymbol{J}}$ is invertible and its inverse can be expressed as

$$
\overline{\boldsymbol{J}}^{-1}=\left[\begin{array}{lll}
\overline{\boldsymbol{J}}_{1}^{M+} & \ldots & \overline{\boldsymbol{J}}_{r}^{M+}
\end{array}\right] .
$$

Proof: This proposition can be proven by construction as follows. Based on (12), if $\overline{\boldsymbol{J}}_{i}$ has full row rank, then $\overline{\boldsymbol{J}}_{i}^{M+}$ exists. Applying (15), one can obtain the result of $\overline{\boldsymbol{J}} \overline{\boldsymbol{J}}^{-1}$ as

$$
\overline{\boldsymbol{J}} \overline{\boldsymbol{J}}^{-1}=\left[\begin{array}{ccc}
\overline{\boldsymbol{J}}_{1} \overline{\boldsymbol{J}}_{1}^{M+} & \ldots & \overline{\boldsymbol{J}}_{1} \overline{\boldsymbol{J}}_{r}^{M+} \\
\vdots & \ddots & \vdots \\
\overline{\boldsymbol{J}}_{r} \overline{\boldsymbol{J}}_{1}^{M+} & \ldots & \overline{\boldsymbol{J}}_{r} \overline{\boldsymbol{J}}_{r}^{M+}
\end{array}\right] .
$$

To prove $\overline{\boldsymbol{J}} \overline{\boldsymbol{J}}^{-1}=\boldsymbol{I}$, it is sufficient to show

case $i=j$ :

$$
\overline{\boldsymbol{J}}_{i} \overline{\boldsymbol{J}}_{j}^{M+}=\overline{\boldsymbol{J}}_{i} \boldsymbol{M}^{-1} \overline{\boldsymbol{J}}_{i}^{T}\left(\overline{\boldsymbol{J}}_{i} \boldsymbol{M}^{-1} \overline{\boldsymbol{J}}_{i}^{T}\right)^{-1}=\boldsymbol{I} .
$$

case $i<j:$ Let $\boldsymbol{\Psi}_{i, j}:=\overline{\boldsymbol{J}}_{i} \boldsymbol{M}^{-1} \overline{\boldsymbol{J}}_{j}^{T}$, then

$$
\overline{\boldsymbol{J}}_{i} \overline{\boldsymbol{J}}_{j}^{M+}=\boldsymbol{\Psi}_{i, j}\left(\overline{\boldsymbol{J}}_{j} \boldsymbol{M}^{-1} \overline{\boldsymbol{J}}_{j}^{T}\right)^{-1} .
$$

For $i=1, \mathbf{\Psi}_{i, j}=\boldsymbol{J}_{1} \boldsymbol{M}^{-1} \boldsymbol{N}_{j} \boldsymbol{J}_{j}^{T}=\mathbf{0}$ due to the dynamic consistency condition (8). Using Lemma 1 for $1<i<j$,

$$
\begin{aligned}
\boldsymbol{\Psi}_{i, j} & =\boldsymbol{J}_{i} \boldsymbol{N}_{i}^{T} \boldsymbol{M}^{-1} \boldsymbol{N}_{j} \boldsymbol{J}_{j}^{T} \\
& =\boldsymbol{J}_{i} \boldsymbol{M}^{-1} \boldsymbol{N}_{j} \boldsymbol{J}_{j}^{T}-\boldsymbol{J}_{i} \boldsymbol{J}_{i-1}^{\text {aug, } \boldsymbol{M +}} \boldsymbol{J}_{i-1}^{\text {aug }} \boldsymbol{M}^{-1} \boldsymbol{N}_{j} \boldsymbol{J}_{j}^{T}
\end{aligned}
$$

Again, (8) leads to $\boldsymbol{J}_{i} \boldsymbol{M}^{-1} \boldsymbol{N}_{j}=\mathbf{0}$ and $\boldsymbol{J}_{i-1}^{\text {aug }} \boldsymbol{M}^{-1} \boldsymbol{N}_{j}=\mathbf{0}$, and thus, $\boldsymbol{\Psi}_{i, j}=\mathbf{0}$.

case $i>j: \boldsymbol{\Psi}_{i, j}=\boldsymbol{\Psi}_{j, i}^{T}=\mathbf{0}$ follows immediately.

\section{APPENDIX B}

The analytical expression of $\boldsymbol{B}$ is derived in the following. Using (5) and (13)-(15), $\dot{\boldsymbol{x}}_{i}$ can be rewritten as

$$
\dot{\boldsymbol{x}}_{i}=\boldsymbol{J}_{i} \dot{\boldsymbol{q}}=\boldsymbol{J}_{i} \overline{\boldsymbol{J}}^{-1} \boldsymbol{v}=\boldsymbol{J}_{i} \sum_{j=1}^{r} \overline{\boldsymbol{J}}_{j}^{\boldsymbol{M +}} \boldsymbol{v}_{j}
$$

for $i=1 \ldots r$. Using the dynamic consistency condition (8), it can be shown that for $i<j \leq r$,

$$
\boldsymbol{J}_{i} \overline{\boldsymbol{J}}_{j}^{M+}=\boldsymbol{J}_{i} \boldsymbol{M}^{-1} \boldsymbol{N}_{j} \boldsymbol{J}_{j}^{T}\left(\overline{\boldsymbol{J}}_{j} \boldsymbol{M}^{-1} \overline{\boldsymbol{J}}_{j}^{T}\right)^{-1}=\mathbf{0} .
$$


Furthermore, since $\boldsymbol{M}^{-1} \boldsymbol{N}_{i}$ is symmetric and $\boldsymbol{N}_{i}$ is idempotent, $\boldsymbol{M}^{-1} \overline{\boldsymbol{J}}_{i}^{T}=\boldsymbol{M}^{-1} \boldsymbol{N}_{i} \overline{\boldsymbol{J}}_{i}^{T}=\boldsymbol{N}_{i}^{T} \boldsymbol{M}^{-1} \overline{\boldsymbol{J}}_{i}^{T}$, and thus,

$$
\boldsymbol{J}_{i} \overline{\boldsymbol{J}}_{i}^{M+}=\boldsymbol{J}_{i} \boldsymbol{N}_{i}^{T} \boldsymbol{M}^{-1} \overline{\boldsymbol{J}}_{i}^{T}\left(\overline{\boldsymbol{J}}_{i} \boldsymbol{M}^{-1} \overline{\boldsymbol{J}}_{i}^{T}\right)^{-1}=\boldsymbol{I} .
$$

Applying (82) and (83) in (81) yields

$$
\boldsymbol{v}_{i}=\dot{\boldsymbol{x}}_{i}-\boldsymbol{J}_{i} \sum_{j=1}^{i-1} \overline{\boldsymbol{J}}_{j}^{M+} \boldsymbol{v}_{j} .
$$

In order to express $\boldsymbol{v}_{i}$ using $\dot{\boldsymbol{x}}_{1} \ldots \dot{\boldsymbol{x}}_{i}$ only, one can utilize the following reformulation

$$
\sum_{j=1}^{i-1} \overline{\boldsymbol{J}}_{j}^{M+} \boldsymbol{v}_{j}=\sum_{j=1}^{i-1} \prod_{k=j+1}^{i-1} \boldsymbol{\Phi}_{k} \overline{\boldsymbol{J}}_{j}^{M+} \dot{\boldsymbol{x}}_{j}
$$

with $\boldsymbol{\Phi}_{k}=\boldsymbol{I}-\overline{\boldsymbol{J}}_{k}^{\boldsymbol{M}+} \boldsymbol{J}_{k}$, which will be shown to hold for $i=$ $2 \ldots r$ by induction. For $i=2, \overline{\boldsymbol{J}}_{1}^{M+} \boldsymbol{v}_{1}=\overline{\boldsymbol{J}}_{1}^{M+} \dot{\boldsymbol{x}}_{1}$ satisfies (85). Suppose that (85) holds for $i=l-1$ with $3<l \leq r$. Then, for $i=l$,

$$
\sum_{j=1}^{l-1} \overline{\boldsymbol{J}}_{j}^{M+} \boldsymbol{v}_{j}=\overline{\boldsymbol{J}}_{l-1}^{M+} \dot{\boldsymbol{x}}_{l-1}+\boldsymbol{\Phi}_{l-1} \sum_{j=1}^{l-2} \overline{\boldsymbol{J}}_{j}^{M+} \boldsymbol{v}_{j}
$$

can be shown by using (84) for $\boldsymbol{v}_{l-1}$. Finally, applying (85) with $i=l-1$ to (86) delivers

$$
\begin{aligned}
\sum_{j=1}^{l-1} \overline{\boldsymbol{J}}_{j}^{M+} \boldsymbol{v}_{j} & =\overline{\boldsymbol{J}}_{l-1}^{M+} \dot{\boldsymbol{x}}_{l-1}+\sum_{j=1}^{l-2} \prod_{k=j+1}^{l-1} \boldsymbol{\Phi}_{k} \overline{\boldsymbol{J}}_{j}^{M+} \dot{\boldsymbol{x}}_{j} \\
& =\sum_{j=1}^{l-1} \prod_{k=j+1}^{l-1} \boldsymbol{\Phi}_{k} \overline{\boldsymbol{J}}_{j}^{M+} \dot{\boldsymbol{x}}_{j},
\end{aligned}
$$

which completes the proof. Combining (84) with (85) yields

$$
\boldsymbol{v}_{i}=\dot{\boldsymbol{x}}_{i}-\boldsymbol{J}_{i} \sum_{j=1}^{i-1} \prod_{k=j+1}^{i-1} \boldsymbol{\Phi}_{k} \overline{\boldsymbol{J}}_{j}^{M+} \dot{\boldsymbol{x}}_{j}
$$

The expressions of $\boldsymbol{B}_{i, j}$ in (21) and (42) follow immediately.

\section{APPENDIX C}

The eigenvalue sensitivity theorem of symmetric matrices from [36] is recapitulated as follows. For the sake of simplicity only the case for minimum eigenvalues is presented.

Theorem 1: [36, Corollary 8.1.6] If $\boldsymbol{A}, \boldsymbol{A}+\boldsymbol{E} \in \mathbb{R}^{n \times n}$ are symmetric, then

$$
\left|\lambda_{\mathrm{m}}(\boldsymbol{A}+\boldsymbol{E})-\lambda_{\mathrm{m}}(\boldsymbol{A})\right| \leq\|\boldsymbol{E}\|
$$

holds, which implies

$$
\lambda_{\mathrm{m}}(\boldsymbol{A}+\boldsymbol{E}) \geq \lambda_{\mathrm{m}}(\boldsymbol{A})-\|\boldsymbol{E}\| .
$$

\section{REFERENCES}

[1] O. Khatib, "A unified approach for motion and force control of robot manipulators: The operational space formulation," IEEE Journal of Robotics and Automation, vol. RA-3, no. 1, pp. 43-53, February 1987.

[2] B. Siciliano and J.-J. Slotine, "A general framework for managing multiple tasks in highly redundant robotic systems," in Proc. of the 5th International Conference on Advanced Robotics, June 1991, pp. 12111216.
[3] O. Khatib, "Inertial properties in robotic manipulation: An object-level framework," International Journal of Robotics Research, vol. 14, no. 1, pp. 19-36, February 1995.

[4] A. Escande, N. Mansard, and P.-B. Wieber, "Hierarchical quadratic programming: Fast online humanoid-robot motion generation," International Journal of Robotics Research, vol. 33, no. 7, pp. 1006-1028, June 2014.

[5] L. Saab, O. E. Ramos, F. Keith, N. Mansard, P. Souères, and J.-I. Fourquet, "Dynamic whole-body motion generation under rigid contacts and other unilateral constraints," IEEE Transactions on Robotics, vol. 29, no. 2, pp. 346-362, April 2013.

[6] A. Herzog, N. Rotella, S. Mason, F. Grimminger, S. Schaal, and L. Righetti, "Momentum control with hierarchical inverse dynamics on a torque-controlled humanoid," Autonomous Robots, vol. 40, no. 3, pp. 473-491, March 2016.

[7] K. Bouyarmane and A. Kheddar, "On weight-prioritized multitask control of humanoid robots," IEEE Transactions on Automatic Control, vol. 63, no. 6, pp. 1632-1647, June 2018.

[8] S. Fahmi, C. Mastalli, M. Focchi, and C. Semini, "Passive whole-body control for quadruped robots: Experimental validation over challenging terrain," IEEE Robotics and Automation Letters, vol. 4, no. 3, pp. 2553 2560, 2019.

[9] O. Khatib, L. Sentis, J. Park, and J. Warren, "Whole-body dynamic behavior and control of human-like robots," International Journal of Humanoid Robotics, vol. 1, no. 1, pp. 29-43, March 2004.

[10] L. Sentis and O. Khatib, "Synthesis of whole-body behaviors through hierarchical control of behavioral primitives," International Journal of Humanoid Robotics, vol. 2, no. 4, pp. 505-518, January 2005.

[11] N. Hogan, "Impedance control: An approach to manipulation: Part I - Theory, Part II - Implementation, Part III - Applications," Journal of Dynamic Systems, Measurement, and Control, vol. 107, pp. 1-24, March 1985.

[12] L. Righetti, J. Buchli, M. Mistry, and S. Schaal, "Inverse dynamics control of floating-base robots with external constraints: A unified view," in Proc. of the 2011 IEEE International Conference on Robotics and Automation, May 2011, pp. 1085-1090.

[13] M. Mistry, J. Buchli, and S. Schaal, "Inverse dynamics control of floating base systems using orthogonal decomposition," in 2010 IEEE International Conference on Robotics and Automation, 2010, pp. 34063412.

[14] C. Ott, A. Kugi, and Y. Nakamura, "Resolving the problem of nonintegrability of nullspace velocities for compliance control of redundant manipulators by using semi-definite lyapunov functions," in Proc. of the 2008 IEEE International Conference on Robotics and Automation, May 2008, pp. 1999-2004.

[15] A. Dietrich, C. Ott, and A. Albu-Schäffer, "Multi-objective compliance control of redundant manipulators: Hierarchy, control, and stability," in Proc. of the 2013 IEEE/RSJ International Conference on Intelligent Robots and Systems, November 2013, pp. 3043-3050.

[16] C. Ott, A. Dietrich, and A. Albu-Schäffer, "Prioritized multi-task compliance control of redundant manipulators," Automatica, vol. 53, pp. 416-423, March 2015.

[17] A. Dietrich and C. Ott, "Hierarchical impedance-based tracking control of kinematically redundant robots," IEEE Transactions on Robotics, vol. 36, no. 1, pp. 204-221, February 2020

[18] G. Antonelli, "Stability analysis for prioritized closed-loop inverse kinematic algorithms for redundant robotic systems," IEEE Transactions on Robotics, vol. 25, no. 5, pp. 985-994, October 2009.

[19] A. Karami, H. Sadeghian, M. Keshmiri, and G. Oriolo, "Hierarchical tracking task control in redundant manipulators with compliance control in the null-space," Mechatronics, vol. 55, pp. 171-179, November 2018.

[20] J. T. Wen, K. Kreutz-Delgado, and D. S. Bayard, "Lyapunov functionbased control laws for revolute robot arms: tracking control, robustness, and adaptive control," IEEE Transactions on Automatic Control, vol. 37, no. 2, pp. 231-237, 1992.

[21] L. L. Whitcomb, A. A. Rizzi, and D. E. Koditschek, "Comparative experiments with a new adaptive controller for robot arms," IEEE Transactions on Robotics and Automation, vol. 9, no. 1, pp. 59-70, February 1993.

[22] V. Santibáñez and R. Kelly, "Strict Lyapunov functions for control of robot manipulators," Automatica, vol. 33, pp. 675-682, 1997.

[23] R. M. Murray, Z. Li, and S. S. Sastry, A Mathematical Introduction to Robotic Manipulation. CRC Press, 1994.

[24] R. Kelly, V. S. Davila, and J. A. L. Perez, Control of Robot Manipulator in Joint Space. Springer-Verlag London, 2005. 
[25] F. Ghorbel, B. Srinivasan, and M. W. Spong, "On the uniform boundedness of the inertia matrix of serial robot manipulators," Journal of Robotic Systems, vol. 15, no. 1, pp. 17-28, 1998.

[26] R. Gunawardana and F. Ghorbel, "On the uniform boundedness of the coriolis/centrifugal terms in the robot equations of motion," International Journal of Robotics and Automation, vol. 14, no. 52, pp. 45-53, 1999.

[27] R. Ortega and M. W. Spong, "Adaptive motion control of rigid robots: A tutorial," Automatica, vol. 25, pp. 877-888, 1989.

[28] S. R. Ploen, "A skew-symmetric form of the recursive newton-euler algorithm for the control of multibody systems," Proceedings of the 1999 American Control Conference, vol. 6, pp. 3770-3773 vol.6, 1999.

[29] P. Chiacchio, S. Chiaverini, L. Sciavicco, and B. Siciliano, "Closed-loop inverse kinematics schemes for constrained redundant manipulators with task space augmentation and task priority strategy," The International Journal of Robotics Research, vol. 10, no. 4, pp. 410-425, 1991.

[30] E. Zergeroglu, D. D. Dawson, I. W. Walker, and P. Setlur, "Nonlinear tracking control of kinematically redundant robot manipulators," IEEE/ASME Transactions on Mechatronics, vol. 9, no. 1, pp. 129-132, March 2004.

[31] H. Sadeghian, L. Villani, M. Keshmiri, and B. Siciliano, "Task-space control of robot manipulators with null-space compliance," IEEE Transactions on Robotics, vol. 30, no. 2, pp. 493-506, April 2014.

[32] A. Dietrich, C. Ott, and A. Albu-Schäffer, "An overview of null space projections for redundant, torque-controlled robots," International Journal of Robotics Research, vol. 34, no. 11, pp. 1385-1400, Sept. 2015.

[33] P. Baerlocher and R. Boulic, "Task-priority formulations for the kinematic control of highly redundant articulated structures," in Proc. of the 1998 IEEE/RSJ International Conference on Intelligent Robots and Systems, October 1998, pp. 323-329.

[34] B. Paden and R. Panja, "Globally asymptotically stable PD+ controller for robot manipulators," International Journal of Control, vol. 47, no. 6 , pp. 1697-1712, 1988.

[35] J. Nakanishi, R. Cory, M. Mistry, J. Peters, and S. Schaal, "Operational space control: A theoretical and empirical comparison," International Journal of Robotics Research, vol. 27, no. 6, pp. 737-757, June 2008.

[36] G. H. Golub and C. F. Van Loan, Matrix Computations. JHU Press, 2013, vol. 3.

[37] J. Duffy, "The fallacy of modern hybrid control theory that is based on "orthogonal complements" of twist and wrench spaces," Journal of Robotic Systems, vol. 7, no. 2, pp. 139-144, 1990.

[38] Q. Lin and J. W. Burdick, "Objective and frame-invariant kinematic metric functions for rigid bodies," The International Journal of Robotics Research, vol. 19, no. 6, pp. 612-625, 2000.

[39] K. L. Doty, C. Melchiorri, and C. Bonivento, "A theory of generalized inverses applied to robotics," International Journal of Robotics Research, vol. 12, no. 1, pp. 1-19, February 1993.

[40] M. Vidyasagar, Nonlinear Systems Analysis. Siam, 2002, vol. 42.

[41] H. K. Khalil, Nonlinear Systems (Third Edition). Prentice Hall, 2002.

[42] S. Boyd and L. Vandenberghe, Convex Optimization. Cambridge University Press, 2004.

[43] A. van der Schaft, L2-Gain and Passivity Techniques in Nonlinear Control, 3rd ed. Springer International Publishing, 2017.

[44] E. D. Sontag and Yuan Wang, "New characterizations of input-to-state stability," IEEE Transactions on Automatic Control, vol. 41, no. 9, pp. 1283-1294, 1996.

[45] E. D. Sontag and Y. Wang, "On characterizations of the input-to-state stability property," Systems \& Control Letters, vol. 24, no. 5, pp. 351359, 1995.

[46] B. R. Barmish and C. M. Lagoa, "The uniform distribution: rigorous justification for its use in robustness analysis," in Proceedings of 35th IEEE Conference on Decision and Control, vol. 3, Dec 1996, pp. 34183423.

[47] A. De Luca and R. Mattone, "Actuator failure detection and isolation using generalized momenta," in 2003 IEEE International Conference on Robotics and Automation, vol. 1, Sep. 2003, pp. 634-639.

[48] A. De Luca, A. Albu-Schaffer, S. Haddadin, and G. Hirzinger, "Collision detection and safe reaction with the DLR-III lightweight manipulator arm," in 2006 IEEE/RSJ International Conference on Intelligent Robots and Systems, Oct 2006, pp. 1623-1630.

[49] F. Caccavale, B. Siciliano, and L. Villani, "Quaternion-based impedance with nondiagonal stiffness for robot manipulators," in Proceedings of the 1998 American Control Conference, vol. 1, 1998, pp. 468-472 vol.1.

[50] A. Albu-Schäffer, C. Ott, U. Frese, and G. Hirzinger, "Cartesian Impedance Control of Redundant Robots: Recent Results with the DLR-Light-Weight-Arms," in Proc. of the 2003 IEEE International Conference on Robotics and Automation, Sept. 2003, pp. 3704-3709.
[51] S. Haddadin, A. De Luca, and A. Albu-Schäffer, "Robot collisions: A survey on detection, isolation, and identification," IEEE Transactions on Robotics, vol. 33, no. 6, pp. 1292-1312, December 2017.

[52] A. Deo and I. Walker, "Overview of Damped Least-Squares Methods for Inverse Kinematics of Robot Manipulators," Journal of Intelligent Robotic Systems, vol. 14, no. 1, pp. 43-68, September 1995.

[53] N. Dehio, D. Kubus, and J. J. Steil, "Continuously shaping projections and operational space tasks," in 2018 IEEE/RSJ International Conference on Intelligent Robots and Systems, 2018, pp. 5995-6002. 